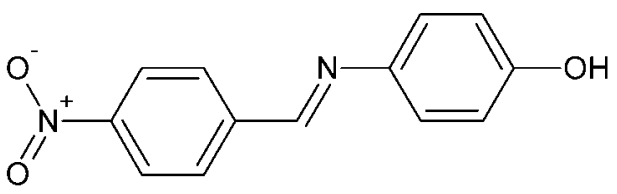

\section{Crystal structure of 4-[(E)-(4-nitro- benzylidene)amino]phenol}

\section{Zeliha Atioğlu, ${ }^{a}$ Mehmet Akkurt, ${ }^{\text {b } *}$ Aliasghar Jarrahpour, Edris Ebrahimi ${ }^{\mathrm{C}}$ and Orhan Büyükgüngör ${ }^{\mathrm{d}}$}

ailke Education and Health Foundation, Cappadocia Vocational College, The Medical Imaging Techniques Program, 50420 Mustafapaşa, Ürgüp, Nevşehir, Turkey, ${ }^{\mathbf{b}}$ Department of Physics, Faculty of Sciences, Erciyes University, 38039 Kayseri, Turkey, ' Department of Chemistry, College of Sciences, Shiraz University 71454 Shiraz, Iran, and ${ }^{\mathbf{d}}$ Department of Physics, Faculty of Arts and Sciences, Ondokuz Mayıs University, 55139 Samsun, Turkey. ${ }^{*}$ Correspondence e-mail: akkurt@erciyes.edu.tr

Received 6 January 2015; accepted 10 January 2015

Edited by W. T. A. Harrison, University of Aberdeen, Scotland

The asymmetric unit of the title compound, $\mathrm{C}_{13} \mathrm{H}_{10} \mathrm{~N}_{2} \mathrm{O}_{3}$, contains four independent molecules (I, II, III and IV). Molecule IV shows whole-molecule disorder over two sets of adjacent sites in a $0.669(10): 0.331$ (10) ratio. The dihedral angles between the aromatic rings are $32.30(13)^{\circ}$ in molecule I, $2.24(14)^{\circ}$ in II, $41.61(13)^{\circ}$ in III, $5.0(5)^{\circ}$ in IV (major component) and $10.2(3)^{\circ}$ in IV (minor component). In the crystal, molecules are linked into layers lying parallel to (024) by $\mathrm{C}-\mathrm{H} \cdots \mathrm{O}$ and $\mathrm{O}-\mathrm{H} \cdots \mathrm{O}$ interactions. The layers interact by $\mathrm{C}-\mathrm{H} \cdots \pi$ and weak aromatic $\pi-\pi$ stacking interactions [centroid-centroid distances $=3.8476(16), 3.725(3)$ and 3.733 (5) ̊].

Keywords: crystal structure; whole-molecule disorder; nitroaromatic compounds; hydrogen bonding; $\mathrm{C}-\mathrm{H} \cdots \pi$ interactions; $\pi-\pi$ stacking interactions.

CCDC reference: 1042888

\section{Related literature}

For background to the importance of Schiff bases and nitroaromatic compounds and their uses, see: Docampo (1990); Ma et al. (2003); Purohit \& Basu (2000); Safwat et al. (1988); Tarafder et al. (2002). For related structures, see: Valkonen et al. (2012); Akkurt et al. (2013, 2014); Atioğlu et al. (2014).

\section{Experimental}

\subsection{Crystal data}

$$
\begin{aligned}
& \mathrm{C}_{13} \mathrm{H}_{10} \mathrm{~N}_{2} \mathrm{O}_{3} \\
& M_{r}=242.23 \\
& \text { Triclinic, } P \overline{1} \\
& a=12.3449(6) \AA \\
& b=13.4266(6) \AA \\
& c=15.7404(7) \AA \\
& \alpha=72.926(4)^{\circ} \\
& \beta=67.390(4)^{\circ}
\end{aligned}
$$

$$
\begin{aligned}
& \gamma=76.824(4)^{\circ} \\
& V=2282.7(2) \AA^{3} \\
& Z=8 \\
& \text { Mo } K \alpha \text { radiation } \\
& \mu=0.10 \mathrm{~mm}^{-1} \\
& T=296 \mathrm{~K} \\
& 0.53 \times 0.49 \times 0.41 \mathrm{~mm}
\end{aligned}
$$

\subsection{Data collection}

Stoe IPDS 2 diffractometer Absorption correction: integration ( $X$-RED32; Stoe \& Cie, 2002)

$T_{\min }=0.952, T_{\max }=0.974$

33476 measured reflections 10470 independent reflections 5234 reflections with $I>2 \sigma(I)$ $R_{\text {int }}=0.099$

\subsection{Refinement}

$R\left[F^{2}>2 \sigma\left(F^{2}\right)\right]=0.070$

$w R\left(F^{2}\right)=0.197$

$S=0.96$

10470 reflections

680 parameters

$$
\begin{aligned}
& 5 \text { restraints } \\
& \text { H-atom parameters constrained } \\
& \Delta \rho_{\max }=0.51 \text { e } \AA^{-3} \\
& \Delta \rho_{\min }=-0.26 \text { e } \AA^{-3}
\end{aligned}
$$

Table 1

Hydrogen-bond geometry $\left(\AA{ }^{\circ}\right)$.

$\mathrm{Cg} 2, \mathrm{Cg} 3, \mathrm{Cg} 4$ and $\mathrm{Cg} 8$ are the centroids of the C8-C13, C14-C19, C21-C26 and $\mathrm{C} 47 \mathrm{~A}-\mathrm{C} 52 \mathrm{~A}$ benzene rings, respectively.

\begin{tabular}{lllll}
\hline$D-\mathrm{H} \cdots A$ & $D-\mathrm{H}$ & $\mathrm{H} \cdots A$ & $D \cdots A$ & $D-\mathrm{H} \cdots A$ \\
\hline $\mathrm{O} 1-\mathrm{H} O 1 \cdots \mathrm{O}^{\mathrm{i}}$ & 0.81 & 2.05 & $2.836(3)$ & 163 \\
$\mathrm{O} 4-\mathrm{H} O 4 \cdots \mathrm{O}^{\mathrm{i}} 2 A^{\mathrm{i}}$ & 0.82 & 2.16 & $2.89(3)$ & 148 \\
$\mathrm{O} 7-\mathrm{H} O 7 \cdots 3^{\text {ii }}$ & 0.82 & 2.06 & $2.832(4)$ & 158 \\
$\mathrm{C} 1-\mathrm{H} 1 \cdots 6^{\mathrm{iii}}$ & 0.93 & 2.53 & $3.417(4)$ & 159 \\
$\mathrm{C} 14-\mathrm{H} 14 \cdots \mathrm{O} 2^{\mathrm{iii}}$ & 0.93 & 2.59 & $3.289(4)$ & 132 \\
$\mathrm{C} 2-\mathrm{H} 2 \cdots C g 2^{\mathrm{iv}}$ & 0.93 & 3.00 & $3.610(3)$ & 125 \\
$\mathrm{C} 15-\mathrm{H} 15 \cdots C g 8^{\mathrm{v}}$ & 0.93 & 2.99 & $3.665(4)$ & 131 \\
$\mathrm{C} 36-\mathrm{H} 36 \cdots C g 3$ & 0.93 & 2.81 & $3.487(3)$ & 130 \\
$\mathrm{C} 43 A-\mathrm{H} 43 A \cdots C g 4^{\mathrm{vi}}$ & 0.93 & 2.80 & $3.539(5)$ & 137 \\
$\mathrm{C} 43-\mathrm{H} 43 \cdots C g 4^{\mathrm{vi}}$ & 0.93 & 2.94 & $3.590(11)$ & 128 \\
\hline Symmetry codes: (i) $x, y-1, z ;(\mathrm{ii})$ & $x, y-1, z+1 ;($ iii) $-x+1,-y+1,-z ;$ (iv) \\
$-x+1,-y, z ;(\mathrm{v})-x+1,-y+1,-z+1 ;(\mathrm{vi})-x, y+1,-z+1$,
\end{tabular}

Data collection: $X$-AREA (Stoe \& Cie, 2002); cell refinement: $X$ $A R E A$; data reduction: $X$-RED32 (Stoe \& Cie, 2002); program(s) used to solve structure: SUPERFLIP (Palatinus \& Chapuis, 2007); program(s) used to refine structure: SHELXL97 (Sheldrick, 2008, 2015); molecular graphics: ORTEP-3 for Windows (Farrugia, 2012); software used to prepare material for publication: Win $G X$ (Farrugia, 2012). 


\section{Acknowledgements}

The authors acknowledge the Faculty of Arts and Sciences, Ondokuz Mayis University, Turkey, for the use of the Stoe IPDS 2 diffractometer (purchased under grant F.279 of the University Research Fund). AJ and EE thank the Shiraz University Research Council for financial support.

Supporting information for this paper is available from the IUCr electronic archives (Reference: HB7349).

\section{References}

Akkurt, M., Jarrahpour, A., Chermahini, M. M., Shiri, P. \& Özdemir, N. (2014). Acta Cryst. E70, o264.
Akkurt, M., Jarrahpour, A., Chermahini, M. M., Shiri, P. \& Tahir, M. N. (2013). Acta Cryst. E69, o247.

Atioğlu, Z., Akkurt, M., Jarrahpour, A., Heiran, R. \& Özdemir, N. (2014). Acta Cryst. E70, o799.

Docampo, R. (1990). Chem. Biol. Interact. 73, 1-27.

Farrugia, L. J. (2012). J. Appl. Cryst. 45, 849-854.

Ma, Y., Fan, Y., Wang, D., Chen, Y. \& Jinan, J. D. X. (2003). Ziran Kexueban, 17, 292-294.

Palatinus, L. \& Chapuis, G. (2007). J. Appl. Cryst. 40, 786-790.

Purohit, V. \& Basu, A. K. (2000). Chem. Res. Toxicol. 13, 673-692.

Safwat, H. M., Ragab, F. A., Eid, N. M. \& Abdel Gawad, M. (1988). Egypt. J. Pharm. Sci. 29, 99-110.

Sheldrick, G. M. (2008). Acta Cryst. A64, 112-122.

Sheldrick, G. M. (2015). Acta Cryst. C71, 3-8.

Stoe \& Cie (2002). $X$-AREA and $X$-RED32. Stoe \& Cie, Darmstadt, Germany. Tarafder, M. T., Kasbollah, A., Saravanan, N., Crouse, K. A., Ali, A. M. \& Tin Oo, K. (2002). J. Biochem. Mol. Biol. Biophys. 6, 85-91.

Valkonen, A., Kolehmainen, E., Grzegórska, A., Ośmiałowski, B., Gawinecki, R. \& Rissanen, K. (2012). Acta Cryst. C68, o279-o282. 


\section{supporting information}

Acta Cryst. (2015). E71, o113-o114 [doi:10.1107/S2056989015000511]

\section{Crystal structure of 4-[(E)-(4-nitrobenzylidene)amino]phenol}

\section{Zeliha Atioğlu, Mehmet Akkurt, Aliasghar Jarrahpour, Edris Ebrahimi and Orhan Büyükgüngör}

\section{S1. Comment}

Schiff bases are potentially biologically active compounds and have been reported to possess antifungal, anticancer, anticonvulsant and diuretic activities (Safwat et al., 1988). Schiff bases derived from various heterocycles were reported to possess cytotoxic activity (Tarafder et al., 2002). It is believed that the presence of a nitro group in the $p$-position appears to be an important condition for the development of bacteriostatic activity (Ma et al., 2003). Nitroaromatic compounds are widely used in medicine, industry and agriculture (Purohit et al., 2000). The anti-malarial activity of nitroaromatic compounds was attributed to the formation of reactive oxygen species during flavoenzyme catalyzed redox cycling reactions and/or oxyhemoglobin oxidation (Docampo, 1990).

The crystal structures of molecules I, II and III of the title compound are ordered, but in (IV) a "whole-disorder molecule" exists (Fig. 1), with the occupancy of the major component being 0.669 (10).

The hydroxy benzene ring is inclined at an angle of $32.30(13)^{\circ}$ in (I), $2.24(14)^{\circ}$ in (II), $41.61(13)^{\circ}$ in (III), $5.0(5)^{\circ}$ in (IV) and $10.2(3)^{\circ}$ in (IVA) with respect to the nitro benzene ring. In the disordered molecules IV, the dihedral angles between the hydroxy benzene rings and and the nitro benzene rings of the two disorders are 2.4 (4) and $3.6(4)^{\circ}$, respectively.

In the asymmetric unit, the bond lengths and bond angles in all molecules are similar and comparable with those of the related structures reported by Valkonen et al., 2012; Akkurt et al., 2014; Atioğlu et al., 2014; Akkurt et al., 2013.

In the crystal structure, intermolecular $\mathrm{C}-\mathrm{H} \cdots \mathrm{O}$ and $\mathrm{O}-\mathrm{H} \cdots \mathrm{O}$ hydrogen bonding interactions link the molecules into a two-dimensional layered structure parallel to the (024) plane (Table 1, Fig. 2). C $-\mathrm{H} \cdots \pi$ interactions and $\pi-\pi$ stacking interactions $[C g 3 \cdots C g 4(1-x, 1-y,-z)=3.8476$ (16) $\AA, C g 6 \cdots C g 7(x, y, z)=3.725$ (3) $\AA$ and $C g 6 \cdots C g 9(x, y, z)=$

3.733 (5) $\AA$; $C g 3, C g 4, C g 6, C g 7$ and $C g 9$ are the centroids of the (C14-C19), (C21-C26), (C34-C39), (C40A-C45A) and $(\mathrm{C} 40-\mathrm{C} 45)$ benzene rings, respectively] between the layers stabilize the molecular packing.

\section{S2. Experimental}

$p$-Nitrobenzaldehyde $(3.0 \mathrm{mmol}, 0.46 \mathrm{~g})$ was added to 4-aminophenol $(3.0 \mathrm{mmol}, 0.33 \mathrm{~g})$ in EtOH $(10 \mathrm{ml})$ refluxed for 4 h. After filtration and recrystallization from EtOH the title Schiff base gave colourless crystals in 85\% yield. Mp: 449 $451 \mathrm{~K} . \mathrm{IR}\left(\mathrm{KBr}, \mathrm{cm}^{-1}\right): 3448(\mathrm{OH}), 1627(\mathrm{C}=\mathrm{N}) .{ }^{1} \mathrm{H}-\mathrm{NMR}\left(250 \mathrm{MHz}, \mathrm{CDCl}_{3}\right), \delta$ (p.p.m.): 6.83 (ArH, 2H, d, J=1.6 Hz), $7.30(\mathrm{ArH}, 2 \mathrm{H}, \mathrm{d}, \mathrm{J}=8.8 \mathrm{~Hz}), 8.13(\mathrm{ArH}, 2 \mathrm{H}, \mathrm{d}, \mathrm{J}=8.8 \mathrm{~Hz}), 8.33(\mathrm{ArH}, 2 \mathrm{H}, \mathrm{d}, \mathrm{J}=8.3 \mathrm{~Hz})$, and $8.80(\mathrm{CH}=\mathrm{N}, 1 \mathrm{H}, \mathrm{s}), 9.66$ (OH, s, 1H). ${ }^{13} \mathrm{C}-\mathrm{NMR}(62.9 \mathrm{MHz}, \mathrm{DMSO}), \delta$ (p.p.m.):116.5, 123.8, 124.6, 129.7, 142.3, 142.8, 149.04, 155.3 (aromatic carbons), $157.9(\mathrm{C}=\mathrm{N})$.

\section{S3. Refinement}

The molecule IV in the asymmetric unit is "whole-molecule disorder" over two sets of sites with occupancies in a ratio of 0.669 (10):0.331 (10). All $\mathrm{H}$ atoms bound to $\mathrm{C}$ atoms and the hydroxyl $\mathrm{H}$ atoms (H10A and H1A0) in the disordered 
molecule IV were positioned geometrically. The $\mathrm{H}$ atoms of the hydroxyl groups in the other molecules were located from electron-density maps, but they were calculated at their idealized positions and all were refined using a riding model with $\mathrm{C}-\mathrm{H}=0.93 \AA, \mathrm{O}-\mathrm{H}=0.82 \AA$ and $U_{\text {iso }}(\mathrm{H})=1.2 U_{\mathrm{eq}}(\mathrm{C})$ and $1.5 U_{\mathrm{eq}}(\mathrm{O})$

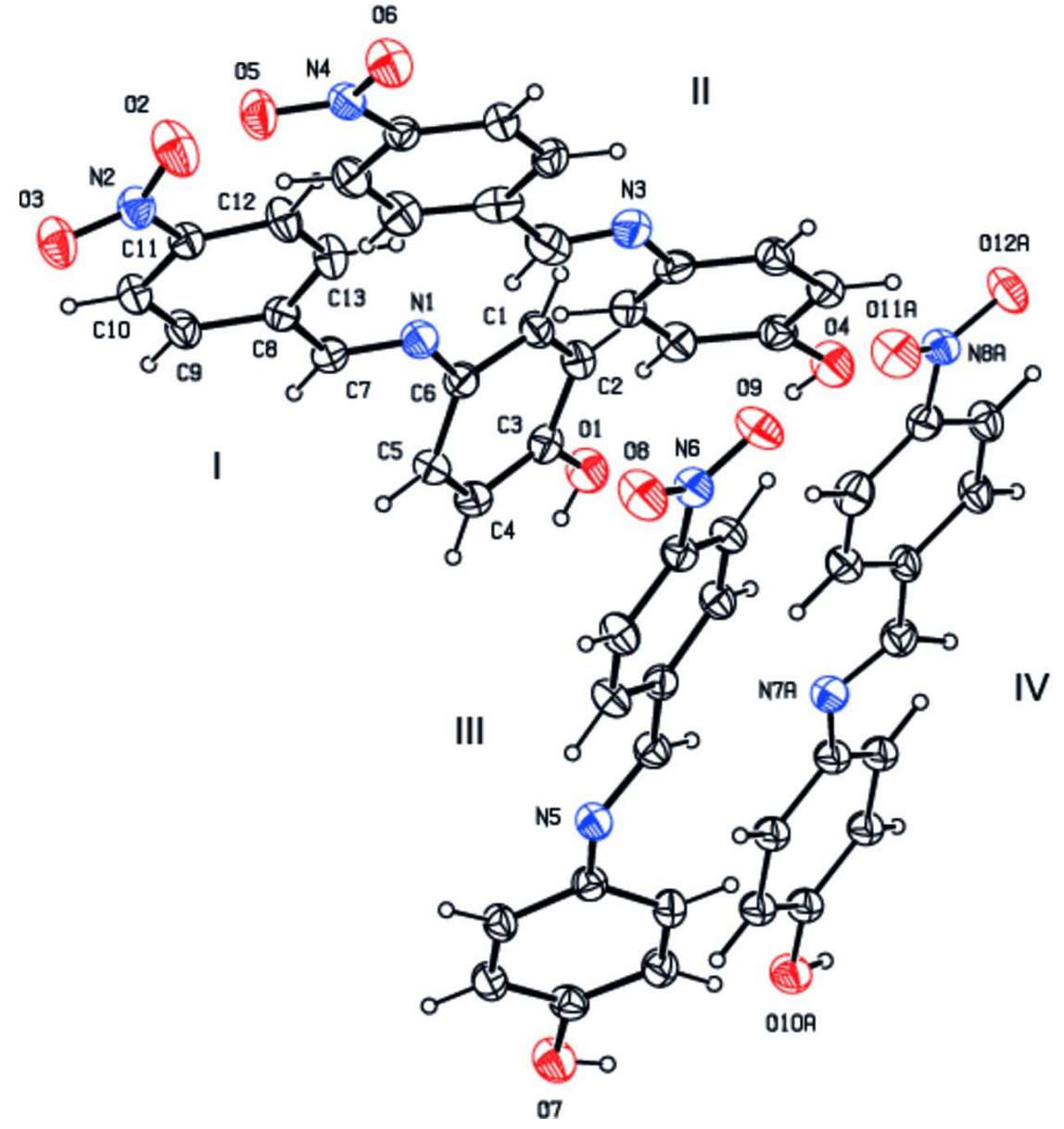

\section{Figure 1}

The molecular structure of the title compound (I) with displacement ellipsoids drawn at the $20 \%$ probability level. Only the major component of the disorder is shown. 


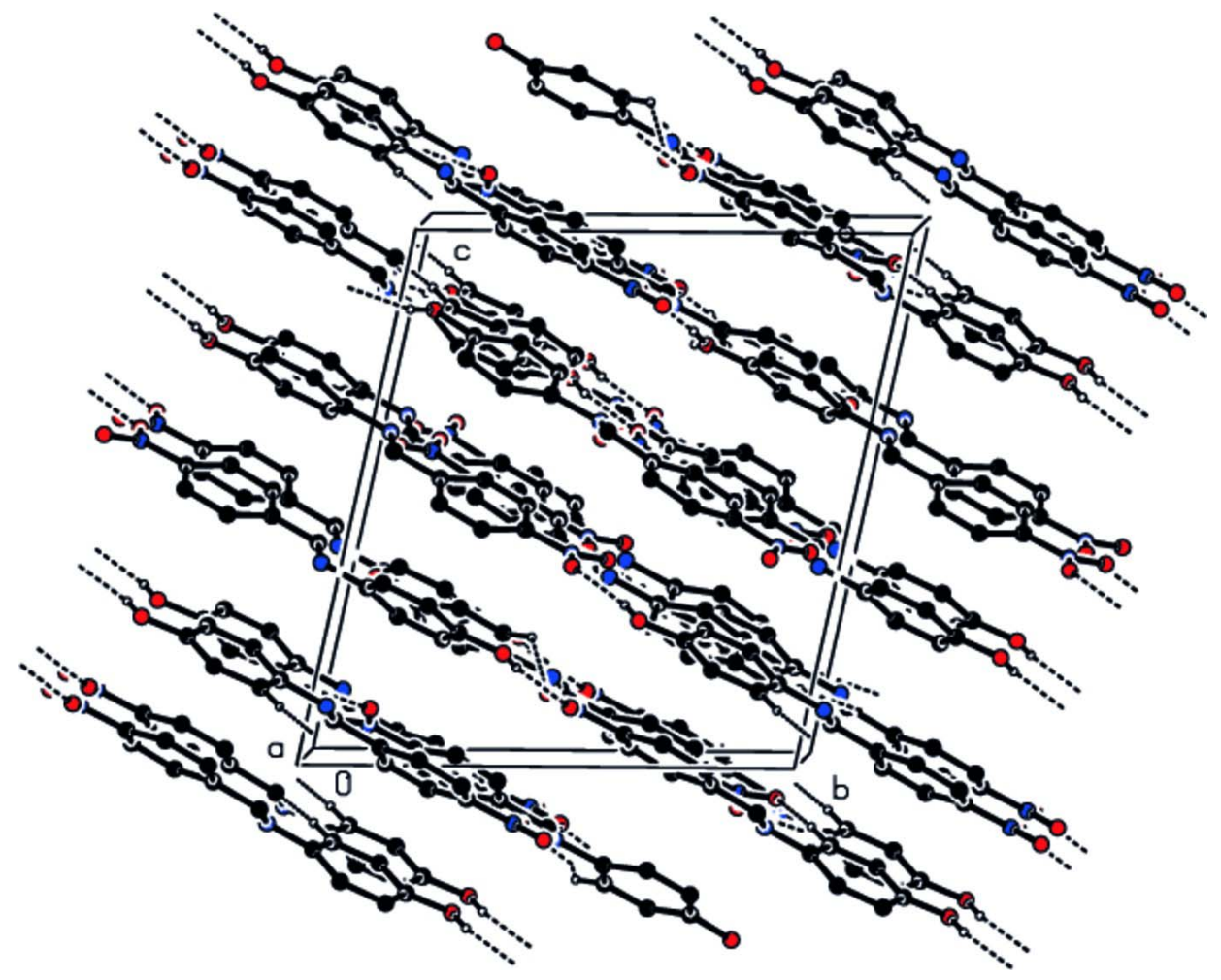

Figure 2

View of the hydrogen bonding and molecular packing of (I) along $a$ axis. Only $\mathrm{H}$ atoms involved in $\mathrm{H}$ bonding and atoms of the major disorder component are shown.

\section{4-[(E)-(4-Nitrobenzylidene)amino]phenol}

\section{Crystal data}

$\mathrm{C}_{13} \mathrm{H}_{10} \mathrm{~N}_{2} \mathrm{O}_{3}$

$M_{r}=242.23$

Triclinic, $P \overline{1}$

Hall symbol: -P 1

$a=12.3449(6) \AA$

$b=13.4266(6) \AA$

$c=15.7404$ (7) $\AA$

$\alpha=72.926(4)^{\circ}$

$\beta=67.390(4)^{\circ}$

$\gamma=76.824(4)^{\circ}$

$V=2282.7(2) \AA^{3}$

\section{Data collection}

Stoe IPDS 2

diffractometer

Radiation source: sealed X-ray tube, $12 \times 0.4$ $\mathrm{mm}$ long-fine focus

Plane graphite monochromator

Detector resolution: 6.67 pixels $\mathrm{mm}^{-1}$

$\omega$ scans

Absorption correction: integration

(X-RED32; Stoe \& Cie, 2002)
$Z=8$

$F(000)=1008$

$D_{\mathrm{x}}=1.410 \mathrm{Mg} \mathrm{m}^{-3}$

Mo $K \alpha$ radiation, $\lambda=0.71073 \AA$

Cell parameters from 25151 reflections

$\theta=1.8-27.9^{\circ}$

$\mu=0.10 \mathrm{~mm}^{-1}$

$T=296 \mathrm{~K}$

Prism, colourless

$0.53 \times 0.49 \times 0.41 \mathrm{~mm}$

$T_{\min }=0.952, T_{\max }=0.974$

33476 measured reflections

10470 independent reflections

5234 reflections with $I>2 \sigma(I)$

$R_{\text {int }}=0.099$

$\theta_{\text {max }}=27.6^{\circ}, \theta_{\text {min }}=1.8^{\circ}$

$h=-16 \rightarrow 15$

$k=-17 \rightarrow 17$

$l=-20 \rightarrow 20$ 


\section{Refinement}

Refinement on $F^{2}$

Least-squares matrix: full

$R\left[F^{2}>2 \sigma\left(F^{2}\right)\right]=0.070$

$w R\left(F^{2}\right)=0.197$

$S=0.96$

10470 reflections

680 parameters

5 restraints

$$
\begin{aligned}
& \text { Hydrogen site location: mixed } \\
& \text { H-atom parameters constrained } \\
& w=1 /\left[\sigma^{2}\left(F_{\mathrm{o}}^{2}\right)+(0.0989 P)^{2}\right] \\
& \text { where } P=\left(F_{\mathrm{o}}^{2}+2 F_{\mathrm{c}}^{2}\right) / 3 \\
& (\Delta / \sigma)_{\max }<0.001 \\
& \Delta \rho_{\max }=0.51 \text { e } \AA^{-3} \\
& \Delta \rho_{\min }=-0.26 \text { e } \AA^{-3}
\end{aligned}
$$

Special details

Geometry. Bond distances, angles etc. have been calculated using the rounded fractional coordinates. All su's are estimated from the variances of the (full) variance-covariance matrix. The cell e.s.d.'s are taken into account in the estimation of distances, angles and torsion angles

\begin{tabular}{|c|c|c|c|c|c|}
\hline & $x$ & $y$ & $z$ & $U_{\text {iso }} * / U_{\text {eq }}$ & Occ. $(<1)$ \\
\hline $\mathrm{O} 1$ & $0.50039(19)$ & $-0.36460(16)$ & $0.28944(16)$ & $0.0835(8)$ & \\
\hline $\mathrm{O} 2$ & $0.1795(2)$ & $0.53357(19)$ & $-0.1317(2)$ & $0.1147(13)$ & \\
\hline $\mathrm{O} 3$ & $0.0208(2)$ & $0.47714(17)$ & -0.10965 (19) & $0.0933(10)$ & \\
\hline N1 & $0.3541(2)$ & $0.04251(18)$ & $0.12063(16)$ & $0.0650(8)$ & \\
\hline $\mathrm{N} 2$ & $0.1187(2)$ & $0.4635(2)$ & $-0.10289(18)$ & $0.0718(9)$ & \\
\hline $\mathrm{C} 1$ & $0.5013(2)$ & $-0.0934(2)$ & $0.15946(19)$ & $0.0620(9)$ & \\
\hline $\mathrm{C} 2$ & $0.5401(2)$ & $-0.1958(2)$ & $0.1993(2)$ & $0.0673(10)$ & \\
\hline $\mathrm{C} 3$ & $0.4587(2)$ & $-0.2649(2)$ & $0.25257(19)$ & $0.0615(9)$ & \\
\hline $\mathrm{C} 4$ & $0.3386(2)$ & $-0.2317(2)$ & $0.26808(19)$ & $0.0645(9)$ & \\
\hline $\mathrm{C} 5$ & $0.3021(2)$ & $-0.1315(2)$ & $0.22690(19)$ & $0.0627(9)$ & \\
\hline C6 & $0.3833(2)$ & $-0.0606(2)$ & $0.16962(18)$ & $0.0587(9)$ & \\
\hline $\mathrm{C} 7$ & $0.2659(2)$ & $0.0614(2)$ & $0.09237(18)$ & $0.0603(9)$ & \\
\hline $\mathrm{C} 8$ & $0.2321(2)$ & $0.1657(2)$ & 0.04025 (18) & $0.0580(9)$ & \\
\hline C9 & $0.1387(2)$ & $0.1805(2)$ & $0.0077(2)$ & $0.0640(10)$ & \\
\hline $\mathrm{C} 10$ & $0.1018(2)$ & $0.2770(2)$ & $-0.0398(2)$ & $0.0652(10)$ & \\
\hline C11 & $0.1614(2)$ & $0.3603(2)$ & -0.05649 (19) & $0.0581(9)$ & \\
\hline $\mathrm{C} 12$ & $0.2590(2)$ & $0.3470(2)$ & $-0.0295(2)$ & $0.0708(10)$ & \\
\hline $\mathrm{C} 13$ & $0.2926(3)$ & $0.2508(2)$ & $0.0190(2)$ & $0.0698(11)$ & \\
\hline $\mathrm{O} 4$ & $0.62408(19)$ & $0.06613(17)$ & $0.33983(17)$ & $0.0884(9)$ & \\
\hline O5 & $0.1439(2)$ & $0.88143(19)$ & $-0.10061(18)$ & $0.0923(9)$ & \\
\hline O6 & $0.2621(2)$ & $0.95766(17)$ & -0.07833 (19) & $0.0932(9)$ & \\
\hline N3 & $0.4627(2)$ & $0.4603(2)$ & $0.15030(18)$ & $0.0831(10)$ & \\
\hline N4 & $0.22268(19)$ & $0.87913(19)$ & $-0.07013(16)$ & $0.0643(8)$ & \\
\hline C14 & $0.5837(2)$ & $0.3448(2)$ & $0.2357(2)$ & $0.0644(9)$ & \\
\hline $\mathrm{C} 15$ & $0.6250(2)$ & $0.2482(2)$ & $0.2825(2)$ & $0.0655(10)$ & \\
\hline $\mathrm{C} 16$ & $0.5807(2)$ & $0.1583(2)$ & $0.29194(19)$ & $0.0639(9)$ & \\
\hline $\mathrm{C} 17$ & $0.4949(2)$ & $0.1654(2)$ & $0.2526(2)$ & $0.0681(10)$ & \\
\hline
\end{tabular}

Refinement. Refinement on $F^{2}$ for ALL reflections except those flagged by the user for potential systematic errors. Weighted $R$-factors $w R$ and all goodnesses of fit $S$ are based on $F^{2}$, conventional $R$-factors $R$ are based on $F$, with $F$ set to zero for negative $F^{2}$. The observed criterion of $F^{2}>\sigma\left(F^{2}\right)$ is used only for calculating - $R$-factor-obs etc. and is not relevant to the choice of reflections for refinement. $R$-factors based on $F^{2}$ are statistically about twice as large as those based on $F$, and $R$-factors based on ALL data will be even larger.

Fractional atomic coordinates and isotropic or equivalent isotropic displacement parameters $\left(\AA^{2}\right)$ 


\begin{tabular}{|c|c|c|c|c|c|}
\hline $\mathrm{C} 18$ & $0.4553(2)$ & $0.2618(2)$ & $0.20622(19)$ & $0.0667(10)$ & \\
\hline C19 & $0.4968(2)$ & $0.3534(2)$ & $0.19782(18)$ & $0.0628(9)$ & \\
\hline $\mathrm{C} 20$ & $0.3792(3)$ & $0.4787(3)$ & $0.1257(3)$ & $0.0897(14)$ & \\
\hline $\mathrm{C} 21$ & $0.3415(3)$ & $0.5865(2)$ & $0.0741(2)$ & $0.0749(11)$ & \\
\hline $\mathrm{C} 22$ & $0.3926(2)$ & $0.6749(3)$ & $0.0635(2)$ & $0.0764(13)$ & \\
\hline $\mathrm{C} 23$ & $0.3554(2)$ & $0.7735(2)$ & $0.01514(19)$ & $0.0649(10)$ & \\
\hline $\mathrm{C} 24$ & $0.2691(2)$ & $0.7782(2)$ & $-0.02215(18)$ & $0.0562(8)$ & \\
\hline $\mathrm{C} 25$ & $0.2217(2)$ & $0.6907(2)$ & $-0.0134(2)$ & $0.0692(11)$ & \\
\hline $\mathrm{C} 26$ & $0.2566(3)$ & $0.5968(3)$ & $0.0359(2)$ & $0.0811(12)$ & \\
\hline $\mathrm{O} 7$ & $-0.10722(19)$ & $-0.36719(16)$ & $0.78633(17)$ & $0.0856(9)$ & \\
\hline O8 & $0.1778(2)$ & $0.53834(18)$ & $0.3923(2)$ & $0.1027(10)$ & \\
\hline O9 & $0.35460(18)$ & $0.47295(17)$ & $0.38559(18)$ & $0.0875(9)$ & \\
\hline N5 & $0.00532(19)$ & $0.04052(17)$ & $0.62254(16)$ & $0.0615(8)$ & \\
\hline N6 & $0.2508(2)$ & $0.46369(18)$ & $0.40531(17)$ & $0.0678(9)$ & \\
\hline $\mathrm{C} 27$ & $0.0410(2)$ & $-0.1359(2)$ & $0.72341(19)$ & $0.0608(9)$ & \\
\hline $\mathrm{C} 28$ & $0.0131(2)$ & $-0.2363(2)$ & $0.7640(2)$ & $0.0670(10)$ & \\
\hline $\mathrm{C} 29$ & $-0.0768(2)$ & $-0.2677(2)$ & $0.74937(19)$ & $0.0616(9)$ & \\
\hline $\mathrm{C} 30$ & $-0.1403(2)$ & $-0.1970(2)$ & $0.6974(2)$ & $0.0662(10)$ & \\
\hline $\mathrm{C} 31$ & $-0.1132(2)$ & $-0.0948(2)$ & $0.65762(19)$ & $0.0595(9)$ & \\
\hline $\mathrm{C} 32$ & $-0.0199(2)$ & $-0.0636(2)$ & $0.66785(18)$ & $0.0560(8)$ & \\
\hline $\mathrm{C} 33$ & $0.1125(2)$ & $0.0584(2)$ & $0.58926(19)$ & $0.0595(9)$ & \\
\hline $\mathrm{C} 34$ & $0.1457(2)$ & $0.1640(2)$ & $0.54077(18)$ & $0.0553(8)$ & \\
\hline $\mathrm{C} 35$ & $0.2643(2)$ & $0.1772(2)$ & $0.49720(19)$ & $0.0605(9)$ & \\
\hline $\mathrm{C} 36$ & $0.3001(2)$ & $0.2740(2)$ & $0.45158(19)$ & $0.0593(9)$ & \\
\hline $\mathrm{C} 37$ & $0.2141(2)$ & $0.3600(2)$ & $0.44933(19)$ & $0.0573(9)$ & \\
\hline C38 & $0.0943(2)$ & $0.3494(2)$ & $0.4904(2)$ & $0.0683(10)$ & \\
\hline C39 & $0.0611(2)$ & $0.2517(2)$ & $0.5364(2)$ & $0.0649(10)$ & \\
\hline O10A & $-0.013(2)$ & $0.073(2)$ & $0.8503(14)$ & $0.077(3)$ & $0.669(10)$ \\
\hline O11A & $0.3512(13)$ & $0.9451(6)$ & $0.4143(11)$ & $0.085(2)$ & $0.669(10)$ \\
\hline O12A & $0.524(2)$ & $0.871(2)$ & $0.408(2)$ & $0.090(3)$ & $0.669(10)$ \\
\hline N7A & $0.1472(4)$ & $0.4600(5)$ & $0.6493(3)$ & $0.0602(18)$ & $0.669(10)$ \\
\hline N8A & $0.416(3)$ & $0.8789(18)$ & $0.4259(18)$ & $0.059(4)$ & $0.669(10)$ \\
\hline $\mathrm{C} 40 \mathrm{~A}$ & $0.20184(19)$ & $0.2548(4)$ & $0.7124(4)$ & $0.0589(9)$ & $0.669(10)$ \\
\hline $\mathrm{C} 41 \mathrm{~A}$ & $0.1522(3)$ & $0.1631(3)$ & $0.7650(4)$ & $0.0589(9)$ & $0.669(10)$ \\
\hline $\mathrm{C} 42 \mathrm{~A}$ & $0.0300(4)$ & $0.1659(2)$ & $0.8035(4)$ & $0.0589(9)$ & $0.669(10)$ \\
\hline $\mathrm{C} 43 \mathrm{~A}$ & -0.04275 (19) & 0.2603 & $0.7893(4)$ & $0.0589(9)$ & $0.669(10)$ \\
\hline $\mathrm{C} 44 \mathrm{~A}$ & $0.0068(3)$ & $0.3520(2)$ & $0.7366(4)$ & $0.0589(9)$ & $0.669(10)$ \\
\hline $\mathrm{C} 45 \mathrm{~A}$ & $0.1291(4)$ & 0.3493 & $0.6982(4)$ & $0.0589(9)$ & $0.669(10)$ \\
\hline $\mathrm{C} 46 \mathrm{~A}$ & $0.2461(5)$ & $0.4821(5)$ & $0.6344(3)$ & $0.0642(17)$ & $0.669(10)$ \\
\hline $\mathrm{C} 47 \mathrm{~A}$ & $0.2851(6)$ & $0.5852(3)$ & $0.5794(2)$ & $0.0548(19)$ & $0.669(10)$ \\
\hline $\mathrm{C} 48 \mathrm{~A}$ & $0.4047(6)$ & $0.5938(3)$ & $0.5513(3)$ & $0.0621(19)$ & $0.669(10)$ \\
\hline C49A & $0.4460(5)$ & $0.6894(4)$ & $0.5002(5)$ & $0.0632(17)$ & $0.669(10)$ \\
\hline $\mathrm{C} 50 \mathrm{~A}$ & $0.3679(6)$ & $0.7764(3)$ & $0.4772(6)$ & $0.071(3)$ & $0.669(10)$ \\
\hline $\mathrm{C} 51 \mathrm{~A}$ & $0.2484(5)$ & $0.7678(4)$ & $0.5053(5)$ & $0.075(3)$ & $0.669(10)$ \\
\hline $\mathrm{C} 52 \mathrm{~A}$ & $0.2070(5)$ & $0.6722(4)$ & $0.5564(4)$ & $0.066(2)$ & $0.669(10)$ \\
\hline $\mathrm{O} 10$ & $-0.009(5)$ & $0.077(5)$ & $0.867(3)$ & $0.077(3)$ & $0.331(10)$ \\
\hline $\mathrm{O} 11$ & $0.339(3)$ & $0.9712(15)$ & $0.410(3)$ & $0.085(2)$ & $0.331(10)$ \\
\hline $\mathrm{O} 12$ & $0.510(5)$ & $0.872(5)$ & $0.396(5)$ & $0.090(3)$ & $0.331(10)$ \\
\hline
\end{tabular}




\begin{tabular}{|c|c|c|c|c|c|}
\hline $\mathrm{N} 7$ & $0.2033(10)$ & $0.4293(8)$ & $0.6474(7)$ & 0.063 & $0.331(10)$ \\
\hline N8 & $0.404(6)$ & $0.862(4)$ & $0.436(4)$ & 0.059 (4) & $0.331(10)$ \\
\hline $\mathrm{C} 40$ & $0.1942(4)$ & $0.2784(7)$ & $0.7093(8)$ & $0.0628(19)$ & $0.331(10)$ \\
\hline $\mathrm{C} 41$ & $0.1664(6)$ & $0.1777(5)$ & $0.7574(9)$ & $0.0628(19)$ & $0.331(10)$ \\
\hline $\mathrm{C} 42$ & $0.0488(7)$ & $0.1596(4)$ & $0.7991(9)$ & $0.0628(19)$ & $0.331(10)$ \\
\hline $\mathrm{C} 43$ & -0.0410 & $0.2423(7)$ & $0.7926(9)$ & $0.0628(19)$ & $0.331(10)$ \\
\hline C44 & $-0.0132(6)$ & $0.3431(5)$ & $0.7445(9)$ & $0.0628(19)$ & $0.331(10)$ \\
\hline $\mathrm{C} 45$ & $0.1044(8)$ & $0.3612(4)$ & $0.7029(8)$ & $0.0628(19)$ & $0.331(10)$ \\
\hline $\mathrm{C} 46$ & $0.1522(7)$ & $0.5239(11)$ & $0.6291(6)$ & $0.054(3)$ & $0.331(10)$ \\
\hline $\mathrm{C} 47$ & $0.2226(11)$ & $0.6094(7)$ & $0.5811(5)$ & $0.054(4)$ & $0.331(10)$ \\
\hline $\mathrm{C} 48$ & $0.3447(11)$ & $0.5931(4)$ & $0.5595(6)$ & $0.062(4)$ & $0.331(10)$ \\
\hline C49 & $0.4103(7)$ & $0.6765(6)$ & $0.5103(8)$ & 0.064 (4) & $0.331(10)$ \\
\hline C50 & $0.3539(7)$ & $0.7762(5)$ & $0.4827(9)$ & 0.034 & $0.331(10)$ \\
\hline C51 & $0.2319(7)$ & $0.7926(6)$ & $0.5043(8)$ & 0.049 & $0.331(10)$ \\
\hline C52 & $0.1662(7)$ & $0.7092(9)$ & $0.5535(6)$ & $0.066(4)$ & $0.331(10)$ \\
\hline H1 & 0.55590 & -0.04590 & 0.12530 & $0.0740^{*}$ & \\
\hline HO1 & 0.44750 & -0.40100 & 0.31640 & $0.1250^{*}$ & \\
\hline $\mathrm{H} 2$ & 0.62010 & -0.21740 & 0.18990 & $0.0810^{*}$ & \\
\hline $\mathrm{H} 4$ & 0.28340 & -0.27760 & 0.30630 & $0.0770^{*}$ & \\
\hline H5 & 0.22190 & -0.11010 & 0.23710 & $0.0750^{*}$ & \\
\hline $\mathrm{H} 7$ & 0.22210 & 0.00750 & 0.10530 & $0.0720^{*}$ & \\
\hline H9 & 0.10040 & 0.12350 & 0.01830 & $0.0770^{*}$ & \\
\hline $\mathrm{H} 10$ & 0.03810 & 0.28650 & -0.06050 & $0.0780^{*}$ & \\
\hline H12 & 0.30080 & 0.40290 & -0.04430 & $0.0850^{*}$ & \\
\hline H13 & 0.35720 & 0.24150 & 0.03850 & $0.0840^{*}$ & \\
\hline $\mathrm{HO} 4$ & 0.58390 & 0.02360 & 0.34280 & $0.1330^{*}$ & \\
\hline H14 & 0.61460 & 0.40460 & 0.22960 & $0.0770^{*}$ & \\
\hline H15 & 0.68310 & 0.24370 & 0.30790 & 0.0790 * & \\
\hline H17 & 0.46490 & 0.10560 & 0.25770 & $0.0820^{*}$ & \\
\hline H18 & 0.39870 & 0.26600 & 0.17940 & $0.0800^{*}$ & \\
\hline $\mathrm{H} 20$ & 0.33560 & 0.42470 & 0.13870 & $0.1080^{*}$ & \\
\hline $\mathrm{H} 22$ & 0.45140 & 0.66760 & 0.08890 & $0.0920^{*}$ & \\
\hline $\mathrm{H} 23$ & 0.38710 & 0.83310 & 0.00830 & $0.0780^{*}$ & \\
\hline $\mathrm{H} 25$ & 0.16580 & 0.69650 & -0.04140 & $0.0830^{*}$ & \\
\hline $\mathrm{H} 26$ & 0.22240 & 0.53820 & 0.04390 & $0.0970^{*}$ & \\
\hline HO7 & -0.06020 & -0.39870 & 0.81320 & $0.1280^{*}$ & \\
\hline $\mathrm{H} 27$ & 0.10170 & -0.11560 & 0.73310 & $0.0730^{*}$ & \\
\hline $\mathrm{H} 28$ & 0.05430 & -0.28330 & 0.80120 & $0.0800^{*}$ & \\
\hline $\mathrm{H} 30$ & -0.20170 & -0.21750 & 0.68880 & $0.0790 *$ & \\
\hline H31 & -0.15790 & -0.04680 & 0.62380 & $0.0710^{*}$ & \\
\hline $\mathrm{H} 33$ & 0.17150 & 0.00280 & 0.59590 & $0.0710^{*}$ & \\
\hline H35 & 0.32130 & 0.11850 & 0.49910 & $0.0730^{*}$ & \\
\hline H36 & 0.38010 & 0.28180 & 0.42290 & $0.0710^{*}$ & \\
\hline $\mathrm{H} 38$ & 0.03760 & 0.40790 & 0.48660 & $0.0820^{*}$ & \\
\hline H39 & -0.01880 & 0.24400 & 0.56500 & $0.0780^{*}$ & \\
\hline H1A0 & 0.04250 & 0.02610 & 0.85250 & $0.1150^{*}$ & $0.669(10)$ \\
\hline $\mathrm{H} 40 \mathrm{~A}$ & 0.28370 & 0.25300 & 0.68660 & $0.0710^{*}$ & $0.669(10)$ \\
\hline H41A & 0.20090 & 0.10000 & 0.77450 & $0.0710^{*}$ & $0.669(10)$ \\
\hline
\end{tabular}


supporting information

\begin{tabular}{llllll} 
H43A & -0.12460 & 0.26210 & 0.81500 & $0.0710^{*}$ & $0.669(10)$ \\
H44A & -0.04180 & 0.41520 & 0.72710 & $0.0710^{*}$ & $0.669(10)$ \\
H46A & 0.29820 & 0.43130 & 0.65890 & $0.0770^{*}$ & $0.669(10)$ \\
H48A & 0.45690 & 0.53560 & 0.56660 & $0.0750^{*}$ & $0.669(10)$ \\
H49A & 0.52600 & 0.69520 & 0.48140 & $0.0760^{*}$ & $0.669(10)$ \\
H51A & 0.19610 & 0.82600 & 0.48990 & $0.0900^{*}$ & $0.669(10)$ \\
H52A & 0.12700 & 0.66640 & 0.57520 & $0.0790^{*}$ & $0.669(10)$ \\
H10A & 0.03870 & 0.02400 & 0.87210 & $0.1150^{*}$ & $0.331(10)$ \\
H40 & 0.27290 & 0.29050 & 0.68140 & $0.0750^{*}$ & $0.331(10)$ \\
H41 & 0.22650 & 0.12230 & 0.76170 & $0.0750^{*}$ & $0.331(10)$ \\
H43 & -0.11970 & 0.23030 & 0.82050 & $0.0750^{*}$ & $0.331(10)$ \\
H44 & -0.07330 & 0.39850 & 0.74020 & $0.0750^{*}$ & $0.331(10)$ \\
H46 & 0.07020 & 0.53720 & 0.64660 & $0.0650^{*}$ & $0.331(10)$ \\
H48 & 0.38240 & 0.52640 & 0.57800 & $0.0750^{*}$ & $0.331(10)$ \\
H49 & 0.49190 & 0.66560 & 0.49580 & $0.0770^{*}$ & $0.331(10)$ \\
H51 & 0.19420 & 0.85930 & 0.48580 & $0.0580^{*}$ & $0.331(10)$ \\
H52 & 0.08460 & 0.72010 & 0.56800 & $0.0800^{*}$ & $0.331(10)$ \\
\hline
\end{tabular}

Atomic displacement parameters $\left(\AA^{2}\right)$

\begin{tabular}{|c|c|c|c|c|c|c|}
\hline & $U^{11}$ & $U^{22}$ & $U^{33}$ & $U^{12}$ & $U^{13}$ & $U^{23}$ \\
\hline $\mathrm{O} 1$ & $0.0828(14)$ & $0.0652(13)$ & $0.0985(16)$ & $-0.0102(10)$ & $-0.0416(12)$ & $0.0020(11)$ \\
\hline $\mathrm{O} 2$ & $0.1063(18)$ & $0.0661(15)$ & $0.179(3)$ & $-0.0303(13)$ & $-0.0768(19)$ & $0.0141(16)$ \\
\hline $\mathrm{O} 3$ & $0.0831(15)$ & $0.0768(15)$ & $0.130(2)$ & $-0.0091(11)$ & $-0.0632(14)$ & $-0.0027(13)$ \\
\hline N1 & $0.0649(13)$ & 0.0607 (14) & $0.0675(14)$ & $-0.0099(10)$ & $-0.0244(11)$ & $-0.0082(11)$ \\
\hline $\mathrm{N} 2$ & $0.0740(16)$ & $0.0640(16)$ & $0.0835(17)$ & $-0.0113(12)$ & $-0.0361(13)$ & $-0.0114(13)$ \\
\hline $\mathrm{C} 1$ & $0.0549(15)$ & $0.0656(17)$ & $0.0673(16)$ & $-0.0179(12)$ & $-0.0255(12)$ & $-0.0038(13)$ \\
\hline $\mathrm{C} 2$ & $0.0544(15)$ & $0.081(2)$ & $0.0685(17)$ & $-0.0084(13)$ & $-0.0273(13)$ & $-0.0117(15)$ \\
\hline $\mathrm{C} 3$ & $0.0712(17)$ & $0.0586(16)$ & $0.0600(15)$ & $-0.0072(13)$ & $-0.0309(13)$ & $-0.0105(13)$ \\
\hline $\mathrm{C} 4$ & $0.0634(16)$ & $0.0664(18)$ & $0.0587(15)$ & $-0.0152(13)$ & $-0.0205(13)$ & $-0.0024(13)$ \\
\hline $\mathrm{C} 5$ & $0.0525(14)$ & $0.0716(18)$ & $0.0614(16)$ & $-0.0138(12)$ & $-0.0143(12)$ & $-0.0139(14)$ \\
\hline C6 & $0.0627(15)$ & $0.0600(16)$ & $0.0555(14)$ & $-0.0134(12)$ & $-0.0220(12)$ & $-0.0096(12)$ \\
\hline $\mathrm{C} 7$ & $0.0608(15)$ & $0.0606(16)$ & $0.0584(15)$ & $-0.0107(12)$ & $-0.0214(12)$ & $-0.0085(13)$ \\
\hline $\mathrm{C} 8$ & $0.0547(14)$ & $0.0623(16)$ & $0.0562(15)$ & $-0.0104(12)$ & $-0.0162(11)$ & $-0.0142(12)$ \\
\hline C9 & $0.0604(15)$ & $0.0621(17)$ & $0.0747(18)$ & $-0.0203(12)$ & $-0.0270(13)$ & $-0.0085(14)$ \\
\hline $\mathrm{C} 10$ & $0.0570(15)$ & $0.0708(19)$ & $0.0754(18)$ & $-0.0112(13)$ & $-0.0295(13)$ & $-0.0162(15)$ \\
\hline C11 & $0.0574(15)$ & $0.0561(16)$ & $0.0613(15)$ & $-0.0074(11)$ & $-0.0213(12)$ & $-0.0128(12)$ \\
\hline $\mathrm{C} 12$ & $0.0655(16)$ & $0.0620(18)$ & $0.100(2)$ & $-0.0155(13)$ & $-0.0395(16)$ & $-0.0199(16)$ \\
\hline $\mathrm{C} 13$ & $0.0675(17)$ & $0.0663(19)$ & $0.090(2)$ & $-0.0060(14)$ & $-0.0434(16)$ & $-0.0184(16)$ \\
\hline $\mathrm{O} 4$ & $0.0802(14)$ & $0.0668(13)$ & $0.1097(17)$ & $-0.0073(10)$ & $-0.0450(13)$ & $0.0074(12)$ \\
\hline O5 & $0.0805(14)$ & $0.0945(17)$ & $0.1134(18)$ & $-0.0065(12)$ & $-0.0596(14)$ & $-0.0084(14)$ \\
\hline O6 & $0.0985(16)$ & $0.0586(13)$ & $0.1221(19)$ & $-0.0195(12)$ & $-0.0481(14)$ & $0.0009(13)$ \\
\hline N3 & $0.0668(15)$ & $0.107(2)$ & $0.0671(15)$ & $-0.0061(14)$ & $-0.0209(13)$ & $-0.0146(15)$ \\
\hline N4 & $0.0567(13)$ & $0.0625(15)$ & $0.0688(14)$ & $-0.0089(11)$ & $-0.0206(11)$ & $-0.0082(12)$ \\
\hline $\mathrm{C} 14$ & $0.0573(15)$ & $0.0617(17)$ & $0.0696(17)$ & $-0.0062(12)$ & $-0.0205(13)$ & $-0.0112(13)$ \\
\hline $\mathrm{C} 15$ & $0.0543(15)$ & $0.0732(19)$ & $0.0655(17)$ & $-0.0113(13)$ & $-0.0179(12)$ & $-0.0121(14)$ \\
\hline $\mathrm{C} 16$ & $0.0575(15)$ & $0.0638(18)$ & $0.0599(15)$ & $-0.0048(13)$ & $-0.0173(12)$ & $-0.0051(13)$ \\
\hline $\mathrm{C} 17$ & $0.0657(16)$ & $0.0669(18)$ & $0.0717(18)$ & -0.0095 & $-0.0232(14)$ & $-0.0157(15)$ \\
\hline
\end{tabular}




\begin{tabular}{|c|c|c|c|c|c|c|}
\hline $\mathrm{C} 18$ & $0.0597(15)$ & $0.077(2)$ & $0.0610(16)$ & $-0.0077(13)$ & $-0.0231(13)$ & $-0.0099(14)$ \\
\hline C19 & $0.0610(15)$ & $0.0600(17)$ & $0.0500(14)$ & $0.0004(12)$ & $-0.0115(12)$ & $-0.0036(12)$ \\
\hline $\mathrm{C} 20$ & $0.072(2)$ & $0.098(3)$ & $0.094(2)$ & $-0.0238(18)$ & $-0.0156(18)$ & $-0.022(2)$ \\
\hline $\mathrm{C} 21$ & $0.081(2)$ & $0.0524(17)$ & $0.0654(18)$ & $-0.0010(14)$ & $-0.0042(15)$ & $-0.0099(14)$ \\
\hline $\mathrm{C} 22$ & $0.0570(16)$ & 0.107 (3) & $0.0600(17)$ & $0.0058(16)$ & $-0.0240(13)$ & $-0.0185(17)$ \\
\hline $\mathrm{C} 23$ & $0.0583(15)$ & $0.0758(19)$ & $0.0648(16)$ & $-0.0127(13)$ & $-0.0230(13)$ & $-0.0163(14)$ \\
\hline $\mathrm{C} 24$ & $0.0530(14)$ & $0.0590(16)$ & $0.0521(14)$ & $-0.0025(11)$ & $-0.0157(11)$ & $-0.0128(12)$ \\
\hline $\mathrm{C} 25$ & $0.0657(16)$ & $0.0652(19)$ & $0.081(2)$ & $-0.0114(14)$ & $-0.0251(15)$ & $-0.0206(15)$ \\
\hline $\mathrm{C} 26$ & $0.079(2)$ & $0.068(2)$ & $0.097(2)$ & $-0.0099(15)$ & $-0.0309(18)$ & $-0.0186(17)$ \\
\hline $\mathrm{O} 7$ & $0.0836(14)$ & $0.0649(13)$ & $0.1052(17)$ & $-0.0191(10)$ & $-0.0400(12)$ & $0.0026(12)$ \\
\hline O8 & $0.0845(15)$ & $0.0623(14)$ & $0.148(2)$ & $-0.0069(11)$ & $-0.0510(15)$ & $0.0085(14)$ \\
\hline O9 & $0.0611(12)$ & $0.0716(14)$ & $0.1182(18)$ & $-0.0193(10)$ & $-0.0186(12)$ & $-0.0131(12)$ \\
\hline N5 & $0.0585(13)$ & $0.0572(14)$ & $0.0683(14)$ & $-0.0084(10)$ & $-0.0236(11)$ & $-0.0102(11)$ \\
\hline N6 & $0.0657(15)$ & $0.0590(15)$ & $0.0754(15)$ & $-0.0043(11)$ & $-0.0259(12)$ & $-0.0109(12)$ \\
\hline $\mathrm{C} 27$ & $0.0576(15)$ & $0.0666(17)$ & $0.0631(16)$ & $-0.0065(12)$ & $-0.0287(13)$ & $-0.0123(13)$ \\
\hline $\mathrm{C} 28$ & $0.0646(16)$ & $0.0671(18)$ & $0.0682(17)$ & $-0.0085(13)$ & $-0.0304(14)$ & $-0.0033(14)$ \\
\hline $\mathrm{C} 29$ & $0.0566(15)$ & $0.0559(16)$ & $0.0638(16)$ & $-0.0101(12)$ & $-0.0159(12)$ & $-0.0056(13)$ \\
\hline $\mathrm{C} 30$ & $0.0583(15)$ & $0.0713(19)$ & $0.0721(18)$ & $-0.0136(13)$ & $-0.0274(13)$ & $-0.0102(15)$ \\
\hline $\mathrm{C} 31$ & $0.0506(13)$ & $0.0600(16)$ & $0.0681(16)$ & $-0.0047(11)$ & $-0.0270(12)$ & $-0.0078(13)$ \\
\hline $\mathrm{C} 32$ & $0.0531(14)$ & $0.0569(16)$ & $0.0568(14)$ & $-0.0056(11)$ & $-0.0186(11)$ & $-0.0125(12)$ \\
\hline $\mathrm{C} 33$ & $0.0571(15)$ & $0.0575(16)$ & $0.0632(16)$ & $-0.0070(12)$ & $-0.0205(12)$ & $-0.0131(13)$ \\
\hline $\mathrm{C} 34$ & $0.0531(14)$ & $0.0598(16)$ & $0.0540(14)$ & $-0.0031(11)$ & $-0.0186(11)$ & $-0.0172(12)$ \\
\hline $\mathrm{C} 35$ & $0.0498(13)$ & $0.0599(16)$ & $0.0704(17)$ & $0.0000(11)$ & $-0.0224(12)$ & $-0.0158(13)$ \\
\hline $\mathrm{C} 36$ & $0.0471(13)$ & $0.0625(17)$ & $0.0654(16)$ & $-0.0031(11)$ & $-0.0165(12)$ & $-0.0171(13)$ \\
\hline $\mathrm{C} 37$ & $0.0589(15)$ & $0.0534(15)$ & $0.0611(15)$ & $-0.0105(11)$ & $-0.0189(12)$ & $-0.0152(12)$ \\
\hline $\mathrm{C} 38$ & $0.0516(14)$ & $0.0597(17)$ & $0.092(2)$ & $0.0066(12)$ & $-0.0254(14)$ & $-0.0243(15)$ \\
\hline C39 & $0.0465(13)$ & $0.0630(17)$ & $0.0822(19)$ & $-0.0069(12)$ & $-0.0166(13)$ & $-0.0199(15)$ \\
\hline O10A & $0.077(2)$ & $0.067(2)$ & $0.081(8)$ & $-0.0226(15)$ & $-0.028(5)$ & $0.002(5)$ \\
\hline O11A & $0.090(4)$ & $0.026(5)$ & $0.123(2)$ & $0.010(4)$ & $-0.044(2)$ & 0.005 \\
\hline $\mathrm{O} 12 \mathrm{~A}$ & $0.060(6)$ & $0.0929(19)$ & $0.122(7)$ & $-0.022(4)$ & $-0.039(3)$ & -0.010 \\
\hline N7A & $0.058(3)$ & $0.046(4)$ & $0.066(2)$ & $-0.003(2)$ & $-0.025(2)$ & $0.005(2)$ \\
\hline N8A & $0.063(6)$ & $0.046(8)$ & $0.066(6)$ & $-0.017(4)$ & $-0.021(5)$ & $-0.003(5)$ \\
\hline $\mathrm{C} 40 \mathrm{~A}$ & $0.0567(16)$ & $0.0599(16)$ & $0.0564(17)$ & $-0.0131(9)$ & $-0.0179(11)$ & $-0.0057(12)$ \\
\hline $\mathrm{C} 41 \mathrm{~A}$ & $0.0567(16)$ & $0.0599(16)$ & $0.0564(17)$ & $-0.0131(9)$ & $-0.0179(11)$ & $-0.0057(12)$ \\
\hline $\mathrm{C} 42 \mathrm{~A}$ & $0.0567(16)$ & $0.0599(16)$ & $0.0564(17)$ & $-0.0131(9)$ & $-0.0179(11)$ & $-0.0057(12)$ \\
\hline $\mathrm{C} 43 \mathrm{~A}$ & $0.0567(16)$ & $0.0599(16)$ & $0.0564(17)$ & $-0.0131(9)$ & $-0.0179(11)$ & $-0.0057(12)$ \\
\hline $\mathrm{C} 44 \mathrm{~A}$ & $0.0567(16)$ & $0.0599(16)$ & $0.0564(17)$ & $-0.0131(9)$ & $-0.0179(11)$ & $-0.0057(12)$ \\
\hline $\mathrm{C} 45 \mathrm{~A}$ & $0.0567(16)$ & $0.0599(16)$ & $0.0564(17)$ & $-0.0131(9)$ & $-0.0179(11)$ & $-0.0057(12)$ \\
\hline $\mathrm{C} 46 \mathrm{~A}$ & $0.067(3)$ & $0.061(3)$ & $0.065(3)$ & $-0.009(2)$ & $-0.028(2)$ & $-0.007(2)$ \\
\hline C47A & $0.042(4)$ & $0.065(3)$ & $0.060(3)$ & $0.004(3)$ & $-0.023(3)$ & $-0.019(2)$ \\
\hline $\mathrm{C} 48 \mathrm{~A}$ & $0.056(4)$ & $0.049(3)$ & $0.083(3)$ & $-0.001(2)$ & $-0.037(3)$ & $-0.004(2)$ \\
\hline C49A & 0.059 & 0.055 & $0.079(3)$ & $-0.007(2)$ & -0.033 & $-0.008(3)$ \\
\hline $\mathrm{C} 50 \mathrm{~A}$ & $0.074(5)$ & $0.095(7)$ & $0.053(5)$ & $-0.028(5)$ & $-0.019(4)$ & $-0.022(4)$ \\
\hline C51A & $0.081(5)$ & $0.062(3)$ & $0.081(5)$ & $0.008(3)$ & $-0.039(4)$ & $-0.014(3)$ \\
\hline $\mathrm{C} 52 \mathrm{~A}$ & 0.053 & $0.075(5)$ & $0.068(3)$ & $-0.010(3)$ & $-0.019(3)$ & $-0.013(3)$ \\
\hline $\mathrm{O} 10$ & $0.077(2)$ & $0.067(2)$ & $0.081(8)$ & $-0.0226(15)$ & $-0.028(5)$ & $0.002(5)$ \\
\hline O11 & $0.090(4)$ & $0.026(5)$ & $0.123(2)$ & $0.010(4)$ & $-0.044(2)$ & 0.005 (4) \\
\hline $\mathrm{O} 12$ & $0.060(6)$ & $0.0929(19)$ & $0.122(7)$ & -0.022 & $-0.039(3)$ & $-0.010(4)$ \\
\hline
\end{tabular}




\begin{tabular}{lllllll}
$\mathrm{N} 7$ & $0.059(7)$ & $0.056(7)$ & $0.082(5)$ & $0.011(5)$ & $-0.042(5)$ & $-0.016(5)$ \\
$\mathrm{N} 8$ & $0.063(6)$ & $0.046(8)$ & $0.066(6)$ & $-0.017(4)$ & $-0.021(5)$ & $-0.003(5)$ \\
C40 & $0.052(3)$ & $0.063(3)$ & $0.070(4)$ & $-0.0075(17)$ & $-0.021(2)$ & $-0.010(3)$ \\
C41 & $0.052(3)$ & $0.063(3)$ & $0.070(4)$ & $-0.0075(17)$ & $-0.021(2)$ & $-0.010(3)$ \\
C42 & $0.052(3)$ & $0.063(3)$ & $0.070(4)$ & $-0.0075(17)$ & $-0.021(2)$ & $-0.010(3)$ \\
C43 & $0.052(3)$ & $0.063(3)$ & $0.070(4)$ & $-0.0075(17)$ & $-0.021(2)$ & $-0.010(3)$ \\
C44 & $0.052(3)$ & $0.063(3)$ & $0.070(4)$ & $-0.0075(17)$ & $-0.021(2)$ & $-0.010(3)$ \\
C45 & $0.052(3)$ & $0.063(3)$ & $0.070(4)$ & $-0.0075(17)$ & $-0.021(2)$ & $-0.010(3)$ \\
C46 & $0.044(4)$ & $0.048(6)$ & $0.059(5)$ & $0.003(4)$ & $-0.018(4)$ & $-0.001(5)$ \\
C47 & $0.040(7)$ & $0.064(8)$ & $0.057(5)$ & $-0.010(6)$ & $-0.012(5)$ & $-0.018(5)$ \\
C48 & $0.028(6)$ & $0.066(6)$ & $0.094(7)$ & $0.017(5)$ & $-0.029(6)$ & $-0.027(5)$ \\
C49 & $0.055(6)$ & $0.061(8)$ & $0.086(8)$ & $-0.009(5)$ & $-0.029(6)$ & $-0.022(6)$ \\
C50 & $0.033(5)$ & $0.012(5)$ & $0.049(7)$ & $0.000(4)$ & $-0.014(4)$ & $0.004(4)$ \\
C51 & $0.031(4)$ & $0.067(6)$ & $0.042(5)$ & $-0.011(4)$ & $-0.005(4)$ & $-0.011(5)$ \\
C52 & $0.059(6)$ & $0.075(9)$ & $0.055(5)$ & $-0.011(6)$ & $-0.013(5)$ & $-0.007(5)$ \\
& & & & & & \\
\hline
\end{tabular}

Geometric parameters $\left(A,{ }^{o}\right)$

\begin{tabular}{|c|c|c|c|}
\hline $\mathrm{O} 1-\mathrm{C} 3$ & $1.361(3)$ & $\mathrm{C} 18-\mathrm{H} 18$ & 0.9300 \\
\hline $\mathrm{O} 2-\mathrm{N} 2$ & $1.210(4)$ & $\mathrm{C} 20-\mathrm{H} 20$ & 0.9300 \\
\hline $\mathrm{O} 3-\mathrm{N} 2$ & $1.222(4)$ & $\mathrm{C} 22-\mathrm{H} 22$ & 0.9300 \\
\hline $\mathrm{O} 1-\mathrm{HO} 1$ & 0.8100 & $\mathrm{C} 23-\mathrm{H} 23$ & 0.9300 \\
\hline $\mathrm{O} 4-\mathrm{C} 16$ & $1.359(4)$ & $\mathrm{C} 25-\mathrm{H} 25$ & 0.9300 \\
\hline $\mathrm{O} 5-\mathrm{N} 4$ & $1.230(4)$ & $\mathrm{C} 26-\mathrm{H} 26$ & 0.9300 \\
\hline $\mathrm{O} 6-\mathrm{N} 4$ & $1.213(4)$ & $\mathrm{C} 27-\mathrm{C} 32$ & $1.395(4)$ \\
\hline $\mathrm{O} 4-\mathrm{HO} 4$ & 0.8200 & $\mathrm{C} 27-\mathrm{C} 28$ & $1.371(4)$ \\
\hline $\mathrm{O} 7-\mathrm{C} 29$ & $1.367(4)$ & $\mathrm{C} 28-\mathrm{C} 29$ & $1.389(4)$ \\
\hline $\mathrm{O} 8-\mathrm{N} 6$ & $1.212(4)$ & $\mathrm{C} 29-\mathrm{C} 30$ & $1.372(4)$ \\
\hline O9-N6 & $1.224(4)$ & $\mathrm{C} 30-\mathrm{C} 31$ & $1.390(4)$ \\
\hline $\mathrm{O} 7-\mathrm{HO} 7$ & 0.8200 & $\mathrm{C} 31-\mathrm{C} 32$ & $1.389(4)$ \\
\hline $\mathrm{O} 10 \mathrm{~A}-\mathrm{C} 42 \mathrm{~A}$ & $1.36(3)$ & $\mathrm{C} 33-\mathrm{C} 34$ & $1.466(4)$ \\
\hline $\mathrm{O} 11 \mathrm{~A}-\mathrm{N} 8 \mathrm{~A}$ & $1.08(3)$ & $\mathrm{C} 34-\mathrm{C} 39$ & $1.389(4)$ \\
\hline $\mathrm{O} 12 \mathrm{~A}-\mathrm{N} 8 \mathrm{~A}$ & $1.24(5)$ & $\mathrm{C} 34-\mathrm{C} 35$ & $1.386(4)$ \\
\hline $\mathrm{O} 10 \mathrm{~A}-\mathrm{H} 1 \mathrm{~A} 0$ & 0.8200 & $\mathrm{C} 35-\mathrm{C} 36$ & $1.368(4)$ \\
\hline $\mathrm{O} 10-\mathrm{C} 42$ & $1.40(6)$ & $\mathrm{C} 36-\mathrm{C} 37$ & $1.381(4)$ \\
\hline $\mathrm{O} 11-\mathrm{N} 8$ & $1.53(7)$ & $\mathrm{C} 37-\mathrm{C} 38$ & $1.390(4)$ \\
\hline $\mathrm{O} 12-\mathrm{N} 8$ & $1.23(10)$ & $\mathrm{C} 38-\mathrm{C} 39$ & $1.368(4)$ \\
\hline $\mathrm{O} 10-\mathrm{H} 10 \mathrm{~A}$ & 0.8200 & $\mathrm{C} 27-\mathrm{H} 27$ & 0.9300 \\
\hline $\mathrm{N} 1-\mathrm{C} 6$ & $1.414(4)$ & $\mathrm{C} 28-\mathrm{H} 28$ & 0.9300 \\
\hline $\mathrm{N} 1-\mathrm{C} 7$ & $1.276(4)$ & $\mathrm{C} 30-\mathrm{H} 30$ & 0.9300 \\
\hline $\mathrm{N} 2-\mathrm{C} 11$ & $1.449(4)$ & C $31-\mathrm{H} 31$ & 0.9300 \\
\hline $\mathrm{N} 3-\mathrm{C} 19$ & $1.461(4)$ & C $33-\mathrm{H} 33$ & 0.9300 \\
\hline $\mathrm{N} 3-\mathrm{C} 20$ & $1.187(5)$ & $\mathrm{C} 35-\mathrm{H} 35$ & 0.9300 \\
\hline $\mathrm{N} 4-\mathrm{C} 24$ & $1.454(4)$ & $\mathrm{C} 36-\mathrm{H} 36$ & 0.9300 \\
\hline $\mathrm{N} 5-\mathrm{C} 32$ & $1.415(4)$ & C $38-\mathrm{H} 38$ & 0.9300 \\
\hline $\mathrm{N} 5-\mathrm{C} 33$ & $1.273(4)$ & C39-H39 & 0.9300 \\
\hline N6-C37 & $1.450(4)$ & $\mathrm{C} 40 \mathrm{~A}-\mathrm{C} 41 \mathrm{~A}$ & $1.390(7)$ \\
\hline $\mathrm{N} 7 \mathrm{~A}-\mathrm{C} 46 \mathrm{~A}$ & $1.240(9)$ & $\mathrm{C} 40 \mathrm{~A}-\mathrm{C} 45 \mathrm{~A}$ & $1.391(7)$ \\
\hline
\end{tabular}




\begin{tabular}{|c|c|c|c|}
\hline $\mathrm{N} 7 \mathrm{~A}-\mathrm{C} 45 \mathrm{~A}$ & $1.483(8)$ & $\mathrm{C} 41 \mathrm{~A}-\mathrm{C} 42 \mathrm{~A}$ & $1.389(7)$ \\
\hline $\mathrm{N} 8 \mathrm{~A}-\mathrm{C} 50 \mathrm{~A}$ & $1.50(3)$ & $\mathrm{C} 42 \mathrm{~A}-\mathrm{C} 43 \mathrm{~A}$ & $1.390(6)$ \\
\hline $\mathrm{N} 7-\mathrm{C} 46$ & $1.286(18)$ & $\mathrm{C} 43 \mathrm{~A}-\mathrm{C} 44 \mathrm{~A}$ & $1.390(6)$ \\
\hline $\mathrm{N} 7-\mathrm{C} 45$ & $1.520(16)$ & $\mathrm{C} 44 \mathrm{~A}-\mathrm{C} 45 \mathrm{~A}$ & $1.390(7)$ \\
\hline $\mathrm{N} 8-\mathrm{C} 50$ & $1.32(6)$ & $\mathrm{C} 46 \mathrm{~A}-\mathrm{C} 47 \mathrm{~A}$ & $1.476(7)$ \\
\hline $\mathrm{C} 1-\mathrm{C} 6$ & $1.383(4)$ & $\mathrm{C} 47 \mathrm{~A}-\mathrm{C} 48 \mathrm{~A}$ & $1.391(10)$ \\
\hline $\mathrm{C} 1-\mathrm{C} 2$ & $1.392(4)$ & $\mathrm{C} 47 \mathrm{~A}-\mathrm{C} 52 \mathrm{~A}$ & $1.390(8)$ \\
\hline $\mathrm{C} 2-\mathrm{C} 3$ & $1.376(4)$ & $\mathrm{C} 48 \mathrm{~A}-\mathrm{C} 49 \mathrm{~A}$ & $1.390(8)$ \\
\hline $\mathrm{C} 3-\mathrm{C} 4$ & $1.393(4)$ & $\mathrm{C} 49 \mathrm{~A}-\mathrm{C} 50 \mathrm{~A}$ & $1.390(9)$ \\
\hline $\mathrm{C} 4-\mathrm{C} 5$ & $1.367(4)$ & $\mathrm{C} 50 \mathrm{~A}-\mathrm{C} 51 \mathrm{~A}$ & $1.390(11)$ \\
\hline $\mathrm{C} 5-\mathrm{C} 6$ & $1.400(4)$ & $\mathrm{C} 51 \mathrm{~A}-\mathrm{C} 52 \mathrm{~A}$ & $1.390(8)$ \\
\hline $\mathrm{C} 7-\mathrm{C} 8$ & $1.460(4)$ & $\mathrm{C} 40 \mathrm{~A}-\mathrm{H} 40 \mathrm{~A}$ & 0.9300 \\
\hline $\mathrm{C} 8-\mathrm{C} 13$ & $1.396(4)$ & $\mathrm{C} 41 \mathrm{~A}-\mathrm{H} 41 \mathrm{~A}$ & 0.9300 \\
\hline $\mathrm{C} 8-\mathrm{C} 9$ & $1.386(4)$ & $\mathrm{C} 43 \mathrm{~A}-\mathrm{H} 43 \mathrm{~A}$ & 0.9300 \\
\hline $\mathrm{C} 9-\mathrm{C} 10$ & $1.367(4)$ & $\mathrm{C} 44 \mathrm{~A}-\mathrm{H} 44 \mathrm{~A}$ & 0.9300 \\
\hline $\mathrm{C} 10-\mathrm{C} 11$ & $1.384(4)$ & $\mathrm{C} 46 \mathrm{~A}-\mathrm{H} 46 \mathrm{~A}$ & 0.9300 \\
\hline $\mathrm{C} 11-\mathrm{C} 12$ & $1.384(4)$ & $\mathrm{C} 48 \mathrm{~A}-\mathrm{H} 48 \mathrm{~A}$ & 0.9300 \\
\hline $\mathrm{C} 12-\mathrm{C} 13$ & $1.358(4)$ & $\mathrm{C} 49 \mathrm{~A}-\mathrm{H} 49 \mathrm{~A}$ & 0.9300 \\
\hline $\mathrm{C} 1-\mathrm{H} 1$ & 0.9300 & $\mathrm{C} 51 \mathrm{~A}-\mathrm{H} 51 \mathrm{~A}$ & 0.9300 \\
\hline $\mathrm{C} 2-\mathrm{H} 2$ & 0.9300 & $\mathrm{C} 52 \mathrm{~A}-\mathrm{H} 52 \mathrm{~A}$ & 0.9300 \\
\hline $\mathrm{C} 4-\mathrm{H} 4$ & 0.9300 & $\mathrm{C} 40-\mathrm{C} 45$ & $1.390(12)$ \\
\hline $\mathrm{C} 5-\mathrm{H} 5$ & 0.9300 & $\mathrm{C} 40-\mathrm{C} 41$ & $1.389(13)$ \\
\hline $\mathrm{C} 7-\mathrm{H} 7$ & 0.9300 & $\mathrm{C} 41-\mathrm{C} 42$ & $1.390(15)$ \\
\hline C9-H9 & 0.9300 & $\mathrm{C} 42-\mathrm{C} 43$ & $1.390(12)$ \\
\hline $\mathrm{C} 10-\mathrm{H} 10$ & 0.9300 & $\mathrm{C} 43-\mathrm{C} 44$ & $1.390(14)$ \\
\hline $\mathrm{C} 12-\mathrm{H} 12$ & 0.9300 & $\mathrm{C} 44-\mathrm{C} 45$ & $1.390(15)$ \\
\hline C13-H13 & 0.9300 & $\mathrm{C} 46-\mathrm{C} 47$ & $1.444(16)$ \\
\hline $\mathrm{C} 14-\mathrm{C} 15$ & $1.382(4)$ & $\mathrm{C} 47-\mathrm{C} 52$ & $1.391(15)$ \\
\hline $\mathrm{C} 14-\mathrm{C} 19$ & $1.384(4)$ & $\mathrm{C} 47-\mathrm{C} 48$ & $1.391(19)$ \\
\hline $\mathrm{C} 15-\mathrm{C} 16$ & $1.383(4)$ & $\mathrm{C} 48-\mathrm{C} 49$ & $1.390(14)$ \\
\hline $\mathrm{C} 16-\mathrm{C} 17$ & $1.392(4)$ & $\mathrm{C} 49-\mathrm{C} 50$ & $1.390(12)$ \\
\hline $\mathrm{C} 17-\mathrm{C} 18$ & $1.371(4)$ & $\mathrm{C} 50-\mathrm{C} 51$ & $1.390(14)$ \\
\hline $\mathrm{C} 18-\mathrm{C} 19$ & $1.390(4)$ & $\mathrm{C} 51-\mathrm{C} 52$ & $1.390(14)$ \\
\hline $\mathrm{C} 20-\mathrm{C} 21$ & $1.505(5)$ & $\mathrm{C} 40-\mathrm{H} 40$ & 0.9300 \\
\hline $\mathrm{C} 21-\mathrm{C} 26$ & $1.360(5)$ & $\mathrm{C} 41-\mathrm{H} 41$ & 0.9300 \\
\hline $\mathrm{C} 21-\mathrm{C} 22$ & $1.407(5)$ & $\mathrm{C} 43-\mathrm{H} 43$ & 0.9300 \\
\hline $\mathrm{C} 22-\mathrm{C} 23$ & $1.394(5)$ & $\mathrm{C} 44-\mathrm{H} 44$ & 0.9300 \\
\hline $\mathrm{C} 23-\mathrm{C} 24$ & $1.383(4)$ & $\mathrm{C} 46-\mathrm{H} 46$ & 0.9300 \\
\hline $\mathrm{C} 24-\mathrm{C} 25$ & $1.378(4)$ & $\mathrm{C} 48-\mathrm{H} 48$ & 0.9300 \\
\hline $\mathrm{C} 25-\mathrm{C} 26$ & $1.346(5)$ & C49-H49 & 0.9300 \\
\hline C14-H14 & 0.9300 & $\mathrm{C} 51-\mathrm{H} 51$ & 0.9300 \\
\hline $\mathrm{C} 15-\mathrm{H} 15$ & 0.9300 & C52-H52 & 0.9300 \\
\hline $\mathrm{C} 17-\mathrm{H} 17$ & 0.9300 & & \\
\hline $\mathrm{C} 3-\mathrm{O} 1-\mathrm{HO} 1$ & 111.00 & $\mathrm{C} 27-\mathrm{C} 32-\mathrm{C} 31$ & $118.1(2)$ \\
\hline $\mathrm{C} 16-\mathrm{O} 4-\mathrm{HO} 4$ & 104.00 & $\mathrm{~N} 5-\mathrm{C} 32-\mathrm{C} 27$ & $124.4(3)$ \\
\hline $\mathrm{C} 29-\mathrm{O} 7-\mathrm{HO} 7$ & 105.00 & $\mathrm{~N} 5-\mathrm{C} 32-\mathrm{C} 31$ & $117.5(2)$ \\
\hline $\mathrm{C} 42 \mathrm{~A}-\mathrm{O} 10 \mathrm{~A}-\mathrm{H} 1 \mathrm{~A} 0$ & 109.00 & $\mathrm{~N} 5-\mathrm{C} 33-\mathrm{C} 34$ & $122.0(3)$ \\
\hline
\end{tabular}




\begin{tabular}{|c|c|c|c|}
\hline $\mathrm{C} 42-\mathrm{O} 10-\mathrm{H} 10 \mathrm{~A}$ & 110.00 & $\mathrm{C} 35-\mathrm{C} 34-\mathrm{C} 39$ & $118.8(2)$ \\
\hline $\mathrm{C} 6-\mathrm{N} 1-\mathrm{C} 7$ & $118.8(2)$ & $\mathrm{C} 33-\mathrm{C} 34-\mathrm{C} 35$ & $119.5(2)$ \\
\hline $\mathrm{O} 2-\mathrm{N} 2-\mathrm{C} 11$ & $119.3(3)$ & $\mathrm{C} 33-\mathrm{C} 34-\mathrm{C} 39$ & $121.7(2)$ \\
\hline $\mathrm{O} 3-\mathrm{N} 2-\mathrm{C} 11$ & $118.5(3)$ & $\mathrm{C} 34-\mathrm{C} 35-\mathrm{C} 36$ & $121.9(3)$ \\
\hline $\mathrm{O} 2-\mathrm{N} 2-\mathrm{O} 3$ & $122.2(3)$ & $\mathrm{C} 35-\mathrm{C} 36-\mathrm{C} 37$ & $118.1(3)$ \\
\hline $\mathrm{C} 19-\mathrm{N} 3-\mathrm{C} 20$ & $119.7(3)$ & $\mathrm{C} 36-\mathrm{C} 37-\mathrm{C} 38$ & $121.6(3)$ \\
\hline $\mathrm{O} 6-\mathrm{N} 4-\mathrm{C} 24$ & $119.3(3)$ & $\mathrm{N} 6-\mathrm{C} 37-\mathrm{C} 36$ & $118.7(2)$ \\
\hline $\mathrm{O} 5-\mathrm{N} 4-\mathrm{O} 6$ & $122.4(3)$ & $\mathrm{N} 6-\mathrm{C} 37-\mathrm{C} 38$ & $119.8(2)$ \\
\hline $\mathrm{O} 5-\mathrm{N} 4-\mathrm{C} 24$ & $118.3(2)$ & $\mathrm{C} 37-\mathrm{C} 38-\mathrm{C} 39$ & $119.1(3)$ \\
\hline $\mathrm{C} 32-\mathrm{N} 5-\mathrm{C} 33$ & $118.8(2)$ & $\mathrm{C} 34-\mathrm{C} 39-\mathrm{C} 38$ & $120.6(3)$ \\
\hline $\mathrm{O} 8-\mathrm{N} 6-\mathrm{C} 37$ & $119.4(3)$ & $\mathrm{C} 32-\mathrm{C} 27-\mathrm{H} 27$ & 119.00 \\
\hline $\mathrm{O} 9-\mathrm{N} 6-\mathrm{C} 37$ & $118.3(2)$ & $\mathrm{C} 28-\mathrm{C} 27-\mathrm{H} 27$ & 119.00 \\
\hline $\mathrm{O} 8-\mathrm{N} 6-\mathrm{O} 9$ & $122.3(3)$ & $\mathrm{C} 29-\mathrm{C} 28-\mathrm{H} 28$ & 120.00 \\
\hline $\mathrm{C} 45 \mathrm{~A}-\mathrm{N} 7 \mathrm{~A}-\mathrm{C} 46 \mathrm{~A}$ & $114.8(6)$ & $\mathrm{C} 27-\mathrm{C} 28-\mathrm{H} 28$ & 120.00 \\
\hline $\mathrm{O} 12 \mathrm{~A}-\mathrm{N} 8 \mathrm{~A}-\mathrm{C} 50 \mathrm{~A}$ & $113(2)$ & $\mathrm{C} 29-\mathrm{C} 30-\mathrm{H} 30$ & 120.00 \\
\hline $\mathrm{O} 11 \mathrm{~A}-\mathrm{N} 8 \mathrm{~A}-\mathrm{C} 50 \mathrm{~A}$ & $115(3)$ & $\mathrm{C} 31-\mathrm{C} 30-\mathrm{H} 30$ & 120.00 \\
\hline $\mathrm{O} 11 \mathrm{~A}-\mathrm{N} 8 \mathrm{~A}-\mathrm{O} 12 \mathrm{~A}$ & $132(3)$ & $\mathrm{C} 30-\mathrm{C} 31-\mathrm{H} 31$ & 120.00 \\
\hline $\mathrm{C} 45-\mathrm{N} 7-\mathrm{C} 46$ & $106.0(10)$ & $\mathrm{C} 32-\mathrm{C} 31-\mathrm{H} 31$ & 120.00 \\
\hline $\mathrm{O} 11-\mathrm{N} 8-\mathrm{O} 12$ & $105(5)$ & $\mathrm{N} 5-\mathrm{C} 33-\mathrm{H} 33$ & 119.00 \\
\hline $\mathrm{O} 11-\mathrm{N} 8-\mathrm{C} 50$ & $126(6)$ & $\mathrm{C} 34-\mathrm{C} 33-\mathrm{H} 33$ & 119.00 \\
\hline $\mathrm{O} 12-\mathrm{N} 8-\mathrm{C} 50$ & $129(6)$ & $\mathrm{C} 36-\mathrm{C} 35-\mathrm{H} 35$ & 119.00 \\
\hline $\mathrm{C} 2-\mathrm{C} 1-\mathrm{C} 6$ & $121.4(3)$ & $\mathrm{C} 34-\mathrm{C} 35-\mathrm{H} 35$ & 119.00 \\
\hline $\mathrm{C} 1-\mathrm{C} 2-\mathrm{C} 3$ & $119.3(3)$ & $\mathrm{C} 35-\mathrm{C} 36-\mathrm{H} 36$ & 121.00 \\
\hline $\mathrm{C} 2-\mathrm{C} 3-\mathrm{C} 4$ & $120.2(3)$ & $\mathrm{C} 37-\mathrm{C} 36-\mathrm{H} 36$ & 121.00 \\
\hline $\mathrm{O} 1-\mathrm{C} 3-\mathrm{C} 4$ & $122.4(3)$ & $\mathrm{C} 37-\mathrm{C} 38-\mathrm{H} 38$ & 120.00 \\
\hline $\mathrm{O} 1-\mathrm{C} 3-\mathrm{C} 2$ & $117.4(3)$ & $\mathrm{C} 39-\mathrm{C} 38-\mathrm{H} 38$ & 121.00 \\
\hline $\mathrm{C} 3-\mathrm{C} 4-\mathrm{C} 5$ & $119.8(3)$ & $\mathrm{C} 38-\mathrm{C} 39-\mathrm{H} 39$ & 120.00 \\
\hline $\mathrm{C} 4-\mathrm{C} 5-\mathrm{C} 6$ & $121.2(3)$ & $\mathrm{C} 34-\mathrm{C} 39-\mathrm{H} 39$ & 120.00 \\
\hline $\mathrm{C} 1-\mathrm{C} 6-\mathrm{C} 5$ & $117.9(2)$ & $\mathrm{C} 41 \mathrm{~A}-\mathrm{C} 40 \mathrm{~A}-\mathrm{C} 45 \mathrm{~A}$ & $120.0(4)$ \\
\hline $\mathrm{N} 1-\mathrm{C} 6-\mathrm{C} 1$ & $117.1(2)$ & $\mathrm{C} 40 \mathrm{~A}-\mathrm{C} 41 \mathrm{~A}-\mathrm{C} 42 \mathrm{~A}$ & $120.0(4)$ \\
\hline $\mathrm{N} 1-\mathrm{C} 6-\mathrm{C} 5$ & $125.0(3)$ & $\mathrm{O} 10 \mathrm{~A}-\mathrm{C} 42 \mathrm{~A}-\mathrm{C} 43 \mathrm{~A}$ & $122.7(12)$ \\
\hline $\mathrm{N} 1-\mathrm{C} 7-\mathrm{C} 8$ & $121.5(3)$ & $\mathrm{C} 41 \mathrm{~A}-\mathrm{C} 42 \mathrm{~A}-\mathrm{C} 43 \mathrm{~A}$ & $120.1(4)$ \\
\hline $\mathrm{C} 9-\mathrm{C} 8-\mathrm{C} 13$ & $118.4(3)$ & $\mathrm{O} 10 \mathrm{~A}-\mathrm{C} 42 \mathrm{~A}-\mathrm{C} 41 \mathrm{~A}$ & $117.1(12)$ \\
\hline $\mathrm{C} 7-\mathrm{C} 8-\mathrm{C} 13$ & $122.6(3)$ & $\mathrm{C} 42 \mathrm{~A}-\mathrm{C} 43 \mathrm{~A}-\mathrm{C} 44 \mathrm{~A}$ & $120.0(4)$ \\
\hline $\mathrm{C} 7-\mathrm{C} 8-\mathrm{C} 9$ & $119.0(2)$ & $\mathrm{C} 43 \mathrm{~A}-\mathrm{C} 44 \mathrm{~A}-\mathrm{C} 45 \mathrm{~A}$ & $120.0(3)$ \\
\hline $\mathrm{C} 8-\mathrm{C} 9-\mathrm{C} 10$ & $121.4(3)$ & $\mathrm{N} 7 \mathrm{~A}-\mathrm{C} 45 \mathrm{~A}-\mathrm{C} 44 \mathrm{~A}$ & $104.2(4)$ \\
\hline $\mathrm{C} 9-\mathrm{C} 10-\mathrm{C} 11$ & $118.4(3)$ & $\mathrm{N} 7 \mathrm{~A}-\mathrm{C} 45 \mathrm{~A}-\mathrm{C} 40 \mathrm{~A}$ & $135.9(5)$ \\
\hline $\mathrm{N} 2-\mathrm{C} 11-\mathrm{C} 12$ & $120.1(2)$ & $\mathrm{C} 40 \mathrm{~A}-\mathrm{C} 45 \mathrm{~A}-\mathrm{C} 44 \mathrm{~A}$ & $120.0(4)$ \\
\hline $\mathrm{C} 10-\mathrm{C} 11-\mathrm{C} 12$ & $121.6(3)$ & $\mathrm{N} 7 \mathrm{~A}-\mathrm{C} 46 \mathrm{~A}-\mathrm{C} 47 \mathrm{~A}$ & $123.1(6)$ \\
\hline $\mathrm{N} 2-\mathrm{C} 11-\mathrm{C} 10$ & $118.3(3)$ & $\mathrm{C} 48 \mathrm{~A}-\mathrm{C} 47 \mathrm{~A}-\mathrm{C} 52 \mathrm{~A}$ & $120.0(4)$ \\
\hline $\mathrm{C} 11-\mathrm{C} 12-\mathrm{C} 13$ & $118.7(3)$ & $\mathrm{C} 46 \mathrm{~A}-\mathrm{C} 47 \mathrm{~A}-\mathrm{C} 52 \mathrm{~A}$ & $122.4(6)$ \\
\hline $\mathrm{C} 8-\mathrm{C} 13-\mathrm{C} 12$ & $121.3(3)$ & $\mathrm{C} 46 \mathrm{~A}-\mathrm{C} 47 \mathrm{~A}-\mathrm{C} 48 \mathrm{~A}$ & $117.6(5)$ \\
\hline $\mathrm{C} 6-\mathrm{C} 1-\mathrm{H} 1$ & 119.00 & $\mathrm{C} 47 \mathrm{~A}-\mathrm{C} 48 \mathrm{~A}-\mathrm{C} 49 \mathrm{~A}$ & $120.0(5)$ \\
\hline $\mathrm{C} 2-\mathrm{C} 1-\mathrm{H} 1$ & 119.00 & $\mathrm{C} 48 \mathrm{~A}-\mathrm{C} 49 \mathrm{~A}-\mathrm{C} 50 \mathrm{~A}$ & $120.1(7)$ \\
\hline $\mathrm{C} 1-\mathrm{C} 2-\mathrm{H} 2$ & 120.00 & $\mathrm{~N} 8 \mathrm{~A}-\mathrm{C} 50 \mathrm{~A}-\mathrm{C} 49 \mathrm{~A}$ & $117.9(15)$ \\
\hline $\mathrm{C} 3-\mathrm{C} 2-\mathrm{H} 2$ & 120.00 & $\mathrm{~N} 8 \mathrm{~A}-\mathrm{C} 50 \mathrm{~A}-\mathrm{C} 51 \mathrm{~A}$ & $122.0(15)$ \\
\hline $\mathrm{C} 3-\mathrm{C} 4-\mathrm{H} 4$ & 120.00 & $\mathrm{C} 49 \mathrm{~A}-\mathrm{C} 50 \mathrm{~A}-\mathrm{C} 51 \mathrm{~A}$ & $120.0(6)$ \\
\hline $\mathrm{C} 5-\mathrm{C} 4-\mathrm{H} 4$ & 120.00 & $\mathrm{C} 50 \mathrm{~A}-\mathrm{C} 51 \mathrm{~A}-\mathrm{C} 52 \mathrm{~A}$ & $120.0(6)$ \\
\hline
\end{tabular}




\begin{tabular}{|c|c|c|c|}
\hline $\mathrm{C} 4-\mathrm{C} 5-\mathrm{H} 5$ & 119.00 & $\mathrm{C} 47 \mathrm{~A}-\mathrm{C} 52 \mathrm{~A}-\mathrm{C} 51 \mathrm{~A}$ & $120.0(6)$ \\
\hline $\mathrm{C} 6-\mathrm{C} 5-\mathrm{H} 5$ & 119.00 & $\mathrm{C} 45 \mathrm{~A}-\mathrm{C} 40 \mathrm{~A}-\mathrm{H} 40 \mathrm{~A}$ & 120.00 \\
\hline $\mathrm{N} 1-\mathrm{C} 7-\mathrm{H} 7$ & 119.00 & $\mathrm{C} 41 \mathrm{~A}-\mathrm{C} 40 \mathrm{~A}-\mathrm{H} 40 \mathrm{~A}$ & 120.00 \\
\hline $\mathrm{C} 8-\mathrm{C} 7-\mathrm{H} 7$ & 119.00 & $\mathrm{C} 40 \mathrm{~A}-\mathrm{C} 41 \mathrm{~A}-\mathrm{H} 41 \mathrm{~A}$ & 120.00 \\
\hline $\mathrm{C} 10-\mathrm{C} 9-\mathrm{H} 9$ & 119.00 & $\mathrm{C} 42 \mathrm{~A}-\mathrm{C} 41 \mathrm{~A}-\mathrm{H} 41 \mathrm{~A}$ & 120.00 \\
\hline $\mathrm{C} 8-\mathrm{C} 9-\mathrm{H} 9$ & 119.00 & $\mathrm{C} 44 \mathrm{~A}-\mathrm{C} 43 \mathrm{~A}-\mathrm{H} 43 \mathrm{~A}$ & 120.00 \\
\hline $\mathrm{C} 9-\mathrm{C} 10-\mathrm{H} 10$ & 121.00 & $\mathrm{C} 42 \mathrm{~A}-\mathrm{C} 43 \mathrm{~A}-\mathrm{H} 43 \mathrm{~A}$ & 120.00 \\
\hline $\mathrm{C} 11-\mathrm{C} 10-\mathrm{H} 10$ & 121.00 & $\mathrm{C} 43 \mathrm{~A}-\mathrm{C} 44 \mathrm{~A}-\mathrm{H} 44 \mathrm{~A}$ & 120.00 \\
\hline $\mathrm{C} 13-\mathrm{C} 12-\mathrm{H} 12$ & 121.00 & $\mathrm{C} 45 \mathrm{~A}-\mathrm{C} 44 \mathrm{~A}-\mathrm{H} 44 \mathrm{~A}$ & 120.00 \\
\hline $\mathrm{C} 11-\mathrm{C} 12-\mathrm{H} 12$ & 121.00 & $\mathrm{C} 47 \mathrm{~A}-\mathrm{C} 46 \mathrm{~A}-\mathrm{H} 46 \mathrm{~A}$ & 118.00 \\
\hline $\mathrm{C} 8-\mathrm{C} 13-\mathrm{H} 13$ & 119.00 & $\mathrm{~N} 7 \mathrm{~A}-\mathrm{C} 46 \mathrm{~A}-\mathrm{H} 46 \mathrm{~A}$ & 118.00 \\
\hline $\mathrm{C} 12-\mathrm{C} 13-\mathrm{H} 13$ & 119.00 & $\mathrm{C} 49 \mathrm{~A}-\mathrm{C} 48 \mathrm{~A}-\mathrm{H} 48 \mathrm{~A}$ & 120.00 \\
\hline $\mathrm{C} 15-\mathrm{C} 14-\mathrm{C} 19$ & $120.6(3)$ & $\mathrm{C} 47 \mathrm{~A}-\mathrm{C} 48 \mathrm{~A}-\mathrm{H} 48 \mathrm{~A}$ & 120.00 \\
\hline $\mathrm{C} 14-\mathrm{C} 15-\mathrm{C} 16$ & $120.7(3)$ & $\mathrm{C} 50 \mathrm{~A}-\mathrm{C} 49 \mathrm{~A}-\mathrm{H} 49 \mathrm{~A}$ & 120.00 \\
\hline $\mathrm{C} 15-\mathrm{C} 16-\mathrm{C} 17$ & $119.4(3)$ & $\mathrm{C} 48 \mathrm{~A}-\mathrm{C} 49 \mathrm{~A}-\mathrm{H} 49 \mathrm{~A}$ & 120.00 \\
\hline $\mathrm{O} 4-\mathrm{C} 16-\mathrm{C} 17$ & $123.0(3)$ & $\mathrm{C} 50 \mathrm{~A}-\mathrm{C} 51 \mathrm{~A}-\mathrm{H} 51 \mathrm{~A}$ & 120.00 \\
\hline $\mathrm{O} 4-\mathrm{C} 16-\mathrm{C} 15$ & $117.6(3)$ & $\mathrm{C} 52 \mathrm{~A}-\mathrm{C} 51 \mathrm{~A}-\mathrm{H} 51 \mathrm{~A}$ & 120.00 \\
\hline $\mathrm{C} 16-\mathrm{C} 17-\mathrm{C} 18$ & $119.1(3)$ & $\mathrm{C} 51 \mathrm{~A}-\mathrm{C} 52 \mathrm{~A}-\mathrm{H} 52 \mathrm{~A}$ & 120.00 \\
\hline $\mathrm{C} 17-\mathrm{C} 18-\mathrm{C} 19$ & $122.3(3)$ & $\mathrm{C} 47 \mathrm{~A}-\mathrm{C} 52 \mathrm{~A}-\mathrm{H} 52 \mathrm{~A}$ & 120.00 \\
\hline $\mathrm{C} 14-\mathrm{C} 19-\mathrm{C} 18$ & $117.9(2)$ & $\mathrm{C} 41-\mathrm{C} 40-\mathrm{C} 45$ & $120.0(8)$ \\
\hline $\mathrm{N} 3-\mathrm{C} 19-\mathrm{C} 18$ & $127.9(3)$ & $\mathrm{C} 40-\mathrm{C} 41-\mathrm{C} 42$ & $120.0(7)$ \\
\hline $\mathrm{N} 3-\mathrm{C} 19-\mathrm{C} 14$ & $114.2(2)$ & $\mathrm{O} 10-\mathrm{C} 42-\mathrm{C} 41$ & $134(3)$ \\
\hline $\mathrm{N} 3-\mathrm{C} 20-\mathrm{C} 21$ & $122.3(4)$ & $\mathrm{C} 41-\mathrm{C} 42-\mathrm{C} 43$ & $120.0(8)$ \\
\hline $\mathrm{C} 20-\mathrm{C} 21-\mathrm{C} 22$ & $122.0(3)$ & $\mathrm{O} 10-\mathrm{C} 42-\mathrm{C} 43$ & $105(3)$ \\
\hline $\mathrm{C} 20-\mathrm{C} 21-\mathrm{C} 26$ & $118.1(3)$ & $\mathrm{C} 42-\mathrm{C} 43-\mathrm{C} 44$ & $120.0(8)$ \\
\hline $\mathrm{C} 22-\mathrm{C} 21-\mathrm{C} 26$ & $120.0(3)$ & $\mathrm{C} 43-\mathrm{C} 44-\mathrm{C} 45$ & $120.0(7)$ \\
\hline $\mathrm{C} 21-\mathrm{C} 22-\mathrm{C} 23$ & $120.3(3)$ & $\mathrm{N} 7-\mathrm{C} 45-\mathrm{C} 44$ & $154.3(8)$ \\
\hline $\mathrm{C} 22-\mathrm{C} 23-\mathrm{C} 24$ & $116.7(3)$ & $\mathrm{C} 40-\mathrm{C} 45-\mathrm{C} 44$ & $120.0(8)$ \\
\hline $\mathrm{N} 4-\mathrm{C} 24-\mathrm{C} 23$ & $119.6(2)$ & $\mathrm{N} 7-\mathrm{C} 45-\mathrm{C} 40$ & $85.7(8)$ \\
\hline $\mathrm{C} 23-\mathrm{C} 24-\mathrm{C} 25$ & $122.6(3)$ & $\mathrm{N} 7-\mathrm{C} 46-\mathrm{C} 47$ & $119.8(10)$ \\
\hline $\mathrm{N} 4-\mathrm{C} 24-\mathrm{C} 25$ & $117.8(2)$ & $\mathrm{C} 46-\mathrm{C} 47-\mathrm{C} 52$ & $118.5(11)$ \\
\hline $\mathrm{C} 24-\mathrm{C} 25-\mathrm{C} 26$ & $119.7(3)$ & $\mathrm{C} 48-\mathrm{C} 47-\mathrm{C} 52$ & $120.0(9)$ \\
\hline $\mathrm{C} 21-\mathrm{C} 26-\mathrm{C} 25$ & $120.8(3)$ & $\mathrm{C} 46-\mathrm{C} 47-\mathrm{C} 48$ & $121.5(9)$ \\
\hline $\mathrm{C} 19-\mathrm{C} 14-\mathrm{H} 14$ & 120.00 & $\mathrm{C} 47-\mathrm{C} 48-\mathrm{C} 49$ & $120.0(8)$ \\
\hline $\mathrm{C} 15-\mathrm{C} 14-\mathrm{H} 14$ & 120.00 & $\mathrm{C} 48-\mathrm{C} 49-\mathrm{C} 50$ & $120.0(10)$ \\
\hline $\mathrm{C} 14-\mathrm{C} 15-\mathrm{H} 15$ & 120.00 & $\mathrm{~N} 8-\mathrm{C} 50-\mathrm{C} 49$ & $127(3)$ \\
\hline $\mathrm{C} 16-\mathrm{C} 15-\mathrm{H} 15$ & 120.00 & $\mathrm{~N} 8-\mathrm{C} 50-\mathrm{C} 51$ & $113(3)$ \\
\hline $\mathrm{C} 18-\mathrm{C} 17-\mathrm{H} 17$ & 120.00 & $\mathrm{C} 49-\mathrm{C} 50-\mathrm{C} 51$ & $120.1(9)$ \\
\hline $\mathrm{C} 16-\mathrm{C} 17-\mathrm{H} 17$ & 120.00 & $\mathrm{C} 50-\mathrm{C} 51-\mathrm{C} 52$ & $120.0(8)$ \\
\hline $\mathrm{C} 19-\mathrm{C} 18-\mathrm{H} 18$ & 119.00 & $\mathrm{C} 47-\mathrm{C} 52-\mathrm{C} 51$ & $120.0(10)$ \\
\hline $\mathrm{C} 17-\mathrm{C} 18-\mathrm{H} 18$ & 119.00 & $\mathrm{C} 41-\mathrm{C} 40-\mathrm{H} 40$ & 120.00 \\
\hline $\mathrm{C} 21-\mathrm{C} 20-\mathrm{H} 20$ & 119.00 & $\mathrm{C} 45-\mathrm{C} 40-\mathrm{H} 40$ & 120.00 \\
\hline $\mathrm{N} 3-\mathrm{C} 20-\mathrm{H} 20$ & 119.00 & $\mathrm{C} 40-\mathrm{C} 41-\mathrm{H} 41$ & 120.00 \\
\hline $\mathrm{C} 23-\mathrm{C} 22-\mathrm{H} 22$ & 120.00 & $\mathrm{C} 42-\mathrm{C} 41-\mathrm{H} 41$ & 120.00 \\
\hline $\mathrm{C} 21-\mathrm{C} 22-\mathrm{H} 22$ & 120.00 & $\mathrm{C} 42-\mathrm{C} 43-\mathrm{H} 43$ & 120.00 \\
\hline $\mathrm{C} 22-\mathrm{C} 23-\mathrm{H} 23$ & 122.00 & $\mathrm{C} 44-\mathrm{C} 43-\mathrm{H} 43$ & 120.00 \\
\hline $\mathrm{C} 24-\mathrm{C} 23-\mathrm{H} 23$ & 122.00 & $\mathrm{C} 43-\mathrm{C} 44-\mathrm{H} 44$ & 120.00 \\
\hline $\mathrm{C} 26-\mathrm{C} 25-\mathrm{H} 25$ & 120.00 & $\mathrm{C} 45-\mathrm{C} 44-\mathrm{H} 44$ & 120.00 \\
\hline
\end{tabular}




\begin{tabular}{|c|c|c|c|}
\hline $\mathrm{C} 24-\mathrm{C} 25-\mathrm{H} 25$ & 120.00 & $\mathrm{~N} 7-\mathrm{C} 46-\mathrm{H} 46$ & 120.00 \\
\hline $\mathrm{C} 25-\mathrm{C} 26-\mathrm{H} 26$ & 120.00 & $\mathrm{C} 47-\mathrm{C} 46-\mathrm{H} 46$ & 120.00 \\
\hline $\mathrm{C} 21-\mathrm{C} 26-\mathrm{H} 26$ & 120.00 & $\mathrm{C} 47-\mathrm{C} 48-\mathrm{H} 48$ & 120.00 \\
\hline $\mathrm{C} 28-\mathrm{C} 27-\mathrm{C} 32$ & $121.3(3)$ & $\mathrm{C} 49-\mathrm{C} 48-\mathrm{H} 48$ & 120.00 \\
\hline $\mathrm{C} 27-\mathrm{C} 28-\mathrm{C} 29$ & $119.8(3)$ & $\mathrm{C} 48-\mathrm{C} 49-\mathrm{H} 49$ & 120.00 \\
\hline $\mathrm{C} 28-\mathrm{C} 29-\mathrm{C} 30$ & $119.9(3)$ & $\mathrm{C} 50-\mathrm{C} 49-\mathrm{H} 49$ & 120.00 \\
\hline $\mathrm{O} 7-\mathrm{C} 29-\mathrm{C} 30$ & $117.5(3)$ & $\mathrm{C} 50-\mathrm{C} 51-\mathrm{H} 51$ & 120.00 \\
\hline $\mathrm{O} 7-\mathrm{C} 29-\mathrm{C} 28$ & $122.6(3)$ & $\mathrm{C} 52-\mathrm{C} 51-\mathrm{H} 51$ & 120.00 \\
\hline $\mathrm{C} 29-\mathrm{C} 30-\mathrm{C} 31$ & $120.2(3)$ & $\mathrm{C} 47-\mathrm{C} 52-\mathrm{H} 52$ & 120.00 \\
\hline $\mathrm{C} 30-\mathrm{C} 31-\mathrm{C} 32$ & $120.6(3)$ & $\mathrm{C} 51-\mathrm{C} 52-\mathrm{H} 52$ & 120.00 \\
\hline $\mathrm{C} 7-\mathrm{N} 1-\mathrm{C} 6-\mathrm{C} 1$ & $149.3(3)$ & $\mathrm{C} 17-\mathrm{C} 18-\mathrm{C} 19-\mathrm{N} 3$ & $179.7(3)$ \\
\hline $\mathrm{C} 7-\mathrm{N} 1-\mathrm{C} 6-\mathrm{C} 5$ & $-30.5(4)$ & $\mathrm{C} 17-\mathrm{C} 18-\mathrm{C} 19-\mathrm{C} 14$ & $2.0(4)$ \\
\hline $\mathrm{C} 6-\mathrm{N} 1-\mathrm{C} 7-\mathrm{C} 8$ & $-179.1(2)$ & $\mathrm{N} 3-\mathrm{C} 20-\mathrm{C} 21-\mathrm{C} 26$ & $170.0(4)$ \\
\hline $\mathrm{O} 2-\mathrm{N} 2-\mathrm{C} 11-\mathrm{C} 10$ & $-168.2(3)$ & $\mathrm{N} 3-\mathrm{C} 20-\mathrm{C} 21-\mathrm{C} 22$ & $-9.0(6)$ \\
\hline $\mathrm{O} 3-\mathrm{N} 2-\mathrm{C} 11-\mathrm{C} 10$ & $13.3(4)$ & $\mathrm{C} 20-\mathrm{C} 21-\mathrm{C} 22-\mathrm{C} 23$ & $179.9(3)$ \\
\hline $\mathrm{O} 2-\mathrm{N} 2-\mathrm{C} 11-\mathrm{C} 12$ & $12.2(4)$ & $\mathrm{C} 22-\mathrm{C} 21-\mathrm{C} 26-\mathrm{C} 25$ & $1.0(5)$ \\
\hline $\mathrm{O} 3-\mathrm{N} 2-\mathrm{C} 11-\mathrm{C} 12$ & $-166.3(3)$ & $\mathrm{C} 26-\mathrm{C} 21-\mathrm{C} 22-\mathrm{C} 23$ & $0.9(5)$ \\
\hline $\mathrm{C} 20-\mathrm{N} 3-\mathrm{C} 19-\mathrm{C} 14$ & $-170.7(3)$ & $\mathrm{C} 20-\mathrm{C} 21-\mathrm{C} 26-\mathrm{C} 25$ & $-178.1(3)$ \\
\hline $\mathrm{C} 20-\mathrm{N} 3-\mathrm{C} 19-\mathrm{C} 18$ & $11.5(5)$ & $\mathrm{C} 21-\mathrm{C} 22-\mathrm{C} 23-\mathrm{C} 24$ & $-1.1(4)$ \\
\hline $\mathrm{C} 19-\mathrm{N} 3-\mathrm{C} 20-\mathrm{C} 21$ & $-178.3(3)$ & $\mathrm{C} 22-\mathrm{C} 23-\mathrm{C} 24-\mathrm{N} 4$ & $177.5(2)$ \\
\hline $\mathrm{O} 5-\mathrm{N} 4-\mathrm{C} 24-\mathrm{C} 23$ & $-178.2(3)$ & $\mathrm{C} 22-\mathrm{C} 23-\mathrm{C} 24-\mathrm{C} 25$ & $-0.5(4)$ \\
\hline $\mathrm{O} 5-\mathrm{N} 4-\mathrm{C} 24-\mathrm{C} 25$ & $-0.2(4)$ & $\mathrm{N} 4-\mathrm{C} 24-\mathrm{C} 25-\mathrm{C} 26$ & $-175.7(3)$ \\
\hline $\mathrm{O} 6-\mathrm{N} 4-\mathrm{C} 24-\mathrm{C} 23$ & $1.4(4)$ & $\mathrm{C} 23-\mathrm{C} 24-\mathrm{C} 25-\mathrm{C} 26$ & $2.4(4)$ \\
\hline $\mathrm{O} 6-\mathrm{N} 4-\mathrm{C} 24-\mathrm{C} 25$ & $179.5(3)$ & $\mathrm{C} 24-\mathrm{C} 25-\mathrm{C} 26-\mathrm{C} 21$ & $-2.6(5)$ \\
\hline $\mathrm{C} 33-\mathrm{N} 5-\mathrm{C} 32-\mathrm{C} 31$ & $-145.5(3)$ & $\mathrm{C} 32-\mathrm{C} 27-\mathrm{C} 28-\mathrm{C} 29$ & $0.5(4)$ \\
\hline $\mathrm{C} 32-\mathrm{N} 5-\mathrm{C} 33-\mathrm{C} 34$ & $179.5(2)$ & $\mathrm{C} 28-\mathrm{C} 27-\mathrm{C} 32-\mathrm{N} 5$ & $-178.9(3)$ \\
\hline $\mathrm{C} 33-\mathrm{N} 5-\mathrm{C} 32-\mathrm{C} 27$ & $35.9(4)$ & $\mathrm{C} 28-\mathrm{C} 27-\mathrm{C} 32-\mathrm{C} 31$ & $2.5(4)$ \\
\hline $\mathrm{O} 9-\mathrm{N} 6-\mathrm{C} 37-\mathrm{C} 38$ & $166.6(3)$ & $\mathrm{C} 27-\mathrm{C} 28-\mathrm{C} 29-\mathrm{O} 7$ & $178.7(3)$ \\
\hline $\mathrm{O} 8-\mathrm{N} 6-\mathrm{C} 37-\mathrm{C} 36$ & $171.0(3)$ & $\mathrm{C} 27-\mathrm{C} 28-\mathrm{C} 29-\mathrm{C} 30$ & $-2.5(4)$ \\
\hline $\mathrm{O} 9-\mathrm{N} 6-\mathrm{C} 37-\mathrm{C} 36$ & $-11.6(4)$ & $\mathrm{C} 28-\mathrm{C} 29-\mathrm{C} 30-\mathrm{C} 31$ & $1.4(4)$ \\
\hline $\mathrm{O} 8-\mathrm{N} 6-\mathrm{C} 37-\mathrm{C} 38$ & $-10.8(4)$ & $\mathrm{O} 7-\mathrm{C} 29-\mathrm{C} 30-\mathrm{C} 31$ & $-179.7(3)$ \\
\hline $\mathrm{C} 45 \mathrm{~A}-\mathrm{N} 7 \mathrm{~A}-\mathrm{C} 46 \mathrm{~A}-\mathrm{C} 47 \mathrm{~A}$ & $174.5(4)$ & $\mathrm{C} 29-\mathrm{C} 30-\mathrm{C} 31-\mathrm{C} 32$ & $1.6(4)$ \\
\hline $\mathrm{C} 46 \mathrm{~A}-\mathrm{N} 7 \mathrm{~A}-\mathrm{C} 45 \mathrm{~A}-\mathrm{C} 44 \mathrm{~A}$ & $159.3(5)$ & $\mathrm{C} 30-\mathrm{C} 31-\mathrm{C} 32-\mathrm{C} 27$ & $-3.5(4)$ \\
\hline $\mathrm{C} 46 \mathrm{~A}-\mathrm{N} 7 \mathrm{~A}-\mathrm{C} 45 \mathrm{~A}-\mathrm{C} 40 \mathrm{~A}$ & $-19.8(9)$ & $\mathrm{C} 30-\mathrm{C} 31-\mathrm{C} 32-\mathrm{N} 5$ & $177.7(2)$ \\
\hline $\mathrm{O} 11 \mathrm{~A}-\mathrm{N} 8 \mathrm{~A}-\mathrm{C} 50 \mathrm{~A}-\mathrm{C} 51 \mathrm{~A}$ & $2(3)$ & $\mathrm{N} 5-\mathrm{C} 33-\mathrm{C} 34-\mathrm{C} 35$ & $-173.2(3)$ \\
\hline $\mathrm{O} 12 \mathrm{~A}-\mathrm{N} 8 \mathrm{~A}-\mathrm{C} 50 \mathrm{~A}-\mathrm{C} 49 \mathrm{~A}$ & $2(3)$ & $\mathrm{N} 5-\mathrm{C} 33-\mathrm{C} 34-\mathrm{C} 39$ & $6.2(4)$ \\
\hline $\mathrm{O} 11 \mathrm{~A}-\mathrm{N} 8 \mathrm{~A}-\mathrm{C} 50 \mathrm{~A}-\mathrm{C} 49 \mathrm{~A}$ & $-175.6(18)$ & $\mathrm{C} 33-\mathrm{C} 34-\mathrm{C} 35-\mathrm{C} 36$ & $-179.6(3)$ \\
\hline $\mathrm{O} 12 \mathrm{~A}-\mathrm{N} 8 \mathrm{~A}-\mathrm{C} 50 \mathrm{~A}-\mathrm{C} 51 \mathrm{~A}$ & $179.1(19)$ & $\mathrm{C} 39-\mathrm{C} 34-\mathrm{C} 35-\mathrm{C} 36$ & $1.1(4)$ \\
\hline $\mathrm{C} 2-\mathrm{C} 1-\mathrm{C} 6-\mathrm{N} 1$ & $-175.4(2)$ & $\mathrm{C} 33-\mathrm{C} 34-\mathrm{C} 39-\mathrm{C} 38$ & $-179.7(3)$ \\
\hline $\mathrm{C} 2-\mathrm{C} 1-\mathrm{C} 6-\mathrm{C} 5$ & $4.3(4)$ & $\mathrm{C} 35-\mathrm{C} 34-\mathrm{C} 39-\mathrm{C} 38$ & $-0.4(4)$ \\
\hline $\mathrm{C} 6-\mathrm{C} 1-\mathrm{C} 2-\mathrm{C} 3$ & $-2.2(4)$ & $\mathrm{C} 34-\mathrm{C} 35-\mathrm{C} 36-\mathrm{C} 37$ & $-0.3(4)$ \\
\hline $\mathrm{C} 1-\mathrm{C} 2-\mathrm{C} 3-\mathrm{C} 4$ & $-1.5(4)$ & $\mathrm{C} 35-\mathrm{C} 36-\mathrm{C} 37-\mathrm{C} 38$ & $-1.2(4)$ \\
\hline $\mathrm{C} 1-\mathrm{C} 2-\mathrm{C} 3-\mathrm{O} 1$ & $179.3(2)$ & $\mathrm{C} 35-\mathrm{C} 36-\mathrm{C} 37-\mathrm{N} 6$ & $177.0(2)$ \\
\hline $\mathrm{C} 2-\mathrm{C} 3-\mathrm{C} 4-\mathrm{C} 5$ & $2.8(4)$ & $\mathrm{C} 36-\mathrm{C} 37-\mathrm{C} 38-\mathrm{C} 39$ & $1.9(4)$ \\
\hline $\mathrm{O} 1-\mathrm{C} 3-\mathrm{C} 4-\mathrm{C} 5$ & $-178.0(3)$ & $\mathrm{N} 6-\mathrm{C} 37-\mathrm{C} 38-\mathrm{C} 39$ & $-176.3(3)$ \\
\hline $\mathrm{C} 3-\mathrm{C} 4-\mathrm{C} 5-\mathrm{C} 6$ & $-0.5(4)$ & $\mathrm{C} 37-\mathrm{C} 38-\mathrm{C} 39-\mathrm{C} 34$ & $-1.1(4)$ \\
\hline $\mathrm{C} 4-\mathrm{C} 5-\mathrm{C} 6-\mathrm{C} 1$ & $-3.0(4)$ & $\mathrm{C} 41 \mathrm{~A}-\mathrm{C} 40 \mathrm{~A}-\mathrm{C} 45 \mathrm{~A}-\mathrm{C} 44 \mathrm{~A}$ & $0.0(8)$ \\
\hline
\end{tabular}




$\begin{array}{llll}\mathrm{C} 4-\mathrm{C} 5-\mathrm{C} 6-\mathrm{N} 1 & 176.7(3) & \mathrm{C} 45 \mathrm{~A}-\mathrm{C} 40 \mathrm{~A}-\mathrm{C} 41 \mathrm{~A}-\mathrm{C} 42 \mathrm{~A} & -0.1(8) \\ \mathrm{N} 1-\mathrm{C} 7-\mathrm{C} 8-\mathrm{C} 13 & -0.7(4) & \mathrm{C} 41 \mathrm{~A}-\mathrm{C} 40 \mathrm{~A}-\mathrm{C} 45 \mathrm{~A}-\mathrm{N} 7 \mathrm{~A} & 179.1(6) \\ \mathrm{N} 1-\mathrm{C} 7-\mathrm{C} 8-\mathrm{C} 9 & 177.2(3) & \mathrm{C} 40 \mathrm{~A}-\mathrm{C} 41 \mathrm{~A}-\mathrm{C} 42 \mathrm{~A}-\mathrm{C} 43 \mathrm{~A} & 0.2(8) \\ \mathrm{C} 9-\mathrm{C} 8-\mathrm{C} 13-\mathrm{C} 12 & 2.3(4) & \mathrm{C} 40 \mathrm{~A}-\mathrm{C} 41 \mathrm{~A}-\mathrm{C} 42 \mathrm{~A}-\mathrm{O} 10 \mathrm{~A} & 177.0(11) \\ \mathrm{C} 7-\mathrm{C} 8-\mathrm{C} 9-\mathrm{C} 10 & 178.6(3) & \mathrm{C} 41 \mathrm{~A}-\mathrm{C} 42 \mathrm{~A}-\mathrm{C} 43 \mathrm{~A}-\mathrm{C} 44 \mathrm{~A} & -0.1(8) \\ \mathrm{C} 13-\mathrm{C} 8-\mathrm{C} 9-\mathrm{C} 10 & -3.4(4) & \mathrm{O} 10 \mathrm{~A}-\mathrm{C} 42 \mathrm{~A}-\mathrm{C} 43 \mathrm{~A}-\mathrm{C} 44 \mathrm{~A} & -176.7(12) \\ \mathrm{C} 7-\mathrm{C} 8-\mathrm{C} 13-\mathrm{C} 12 & -179.9(3) & \mathrm{C} 42 \mathrm{~A}-\mathrm{C} 43 \mathrm{~A}-\mathrm{C} 44 \mathrm{~A}-\mathrm{C} 45 \mathrm{~A} & -0.1(8) \\ \mathrm{C} 8-\mathrm{C} 9-\mathrm{C} 10-\mathrm{C} 11 & 1.1(4) & \mathrm{C} 43 \mathrm{~A}-\mathrm{C} 44 \mathrm{~A}-\mathrm{C} 45 \mathrm{~A}-\mathrm{N} 7 \mathrm{~A} & -179.2(5) \\ \mathrm{C} 9-\mathrm{C} 10-\mathrm{C} 11-\mathrm{N} 2 & -177.1(3) & \mathrm{C} 43 \mathrm{~A}-\mathrm{C} 44 \mathrm{~A}-\mathrm{C} 45 \mathrm{~A}-\mathrm{C} 40 \mathrm{~A} & 0.1(8) \\ \mathrm{C} 9-\mathrm{C} 10-\mathrm{C} 11-\mathrm{C} 12 & 2.4(4) & \mathrm{N} 7 \mathrm{~A}-\mathrm{C} 46 \mathrm{~A}-\mathrm{C} 47 \mathrm{~A}-\mathrm{C} 48 \mathrm{~A} & -167.3(5) \\ \mathrm{N} 2-\mathrm{C} 11-\mathrm{C} 12-\mathrm{C} 13 & 176.0(3) & \mathrm{N} 7 \mathrm{~A}-\mathrm{C} 46 \mathrm{~A}-\mathrm{C} 47 \mathrm{~A}-\mathrm{C} 52 \mathrm{~A} & 13.4(7) \\ \mathrm{C} 10-\mathrm{C} 11-\mathrm{C} 12-\mathrm{C} 13 & -3.6(4) & \mathrm{C} 46 \mathrm{~A}-\mathrm{C} 47 \mathrm{~A}-\mathrm{C} 48 \mathrm{~A}-\mathrm{C} 49 \mathrm{~A} & -179.3(5) \\ \mathrm{C} 11-\mathrm{C} 12-\mathrm{C} 13-\mathrm{C} 8 & 1.2(4) & \mathrm{C} 52 \mathrm{~A}-\mathrm{C} 47 \mathrm{~A}-\mathrm{C} 48 \mathrm{~A}-\mathrm{C} 49 \mathrm{~A} & 0.0(7) \\ \mathrm{C} 19-\mathrm{C} 14-\mathrm{C} 15-\mathrm{C} 16 & 0.3(4) & \mathrm{C} 46 \mathrm{~A}-\mathrm{C} 47 \mathrm{~A}-\mathrm{C} 52 \mathrm{~A}-\mathrm{C} 51 \mathrm{~A} & 179.3(5) \\ \mathrm{C} 15-\mathrm{C} 14-\mathrm{C} 19-\mathrm{N} 3 & -179.8(3) & \mathrm{C} 48 \mathrm{~A}-\mathrm{C} 47 \mathrm{~A}-\mathrm{C} 52 \mathrm{~A}-\mathrm{C} 51 \mathrm{~A} & 0.0(7) \\ \mathrm{C} 15-\mathrm{C} 14-\mathrm{C} 19-\mathrm{C} 18 & -1.7(4) & \mathrm{C} 47 \mathrm{~A}-\mathrm{C} 48 \mathrm{~A}-\mathrm{C} 49 \mathrm{~A}-\mathrm{C} 50 \mathrm{~A} & 0.0(9) \\ \mathrm{C} 14-\mathrm{C} 15-\mathrm{C} 16-\mathrm{C} 17 & 0.9(4) & \mathrm{C} 48 \mathrm{~A}-\mathrm{C} 49 \mathrm{~A}-\mathrm{C} 50 \mathrm{~A}-\mathrm{N} 8 \mathrm{~A} & 177.2(12) \\ \mathrm{C} 14-\mathrm{C} 15-\mathrm{C} 16-\mathrm{O} 4 & -179.2(3) & \mathrm{C} 48 \mathrm{~A}-\mathrm{C} 49 \mathrm{~A}-\mathrm{C} 50 \mathrm{~A}-\mathrm{C} 51 \mathrm{~A} & 0.0(11) \\ \mathrm{C} 15-\mathrm{C} 16-\mathrm{C} 17-\mathrm{C} 18 & -0.7(4) & \mathrm{N} 8 \mathrm{~A}-\mathrm{C} 50 \mathrm{~A}-\mathrm{C} 51 \mathrm{~A}-\mathrm{C} 52 \mathrm{~A} & -177.1(13) \\ \mathrm{O} 4-\mathrm{C} 16-\mathrm{C} 17-\mathrm{C} 18 & 179.4(3) & \mathrm{C} 49 \mathrm{~A}-\mathrm{C} 50 \mathrm{~A}-\mathrm{C} 51 \mathrm{~A}-\mathrm{C} 52 \mathrm{~A} & 0.0(11) \\ \mathrm{C} 16-\mathrm{C} 17-\mathrm{C} 18-\mathrm{C} 19 & -0.7(4) & \mathrm{C} 50 \mathrm{~A}-\mathrm{C} 51 \mathrm{~A}-\mathrm{C} 52 \mathrm{~A}-\mathrm{C} 47 \mathrm{~A} & 0.0(9) \\ \end{array}$

Hydrogen-bond geometry $\left(A,{ }^{\circ}\right)$

$\mathrm{Cg} 2, \mathrm{Cg} 3, \mathrm{Cg} 4$ and $\mathrm{Cg} 8$ are the centroids of the $\mathrm{C} 8-\mathrm{C} 13, \mathrm{C} 14-\mathrm{C} 19, \mathrm{C} 21-\mathrm{C} 26$ and $\mathrm{C} 47 A-\mathrm{C} 52 A$ benzene rings, respectively.

\begin{tabular}{lllll}
\hline$D-\mathrm{H} \cdots A$ & $D-\mathrm{H}$ & $\mathrm{H} \cdots A$ & $D \cdots A$ & $D-\mathrm{H}^{\cdots} A A$ \\
\hline $\mathrm{O} 1-\mathrm{HO} 1 \cdots \mathrm{O} 9^{\mathrm{i}}$ & 0.81 & 2.05 & $2.836(3)$ & 163 \\
$\mathrm{O} 4-\mathrm{HO} 4 \cdots \mathrm{O} 12 A^{\mathrm{i}}$ & 0.82 & 2.16 & $2.89(3)$ & 148 \\
$\mathrm{O} 7-\mathrm{HO} 7 \cdots 3^{\mathrm{ii}}$ & 0.82 & 2.06 & $2.832(4)$ & 158 \\
$\mathrm{C} 1-\mathrm{H} 1 \cdots 6^{\mathrm{iii}}$ & 0.93 & 2.53 & $3.417(4)$ & 159 \\
$\mathrm{C} 14-\mathrm{H} 14 \cdots \mathrm{O} 2^{\mathrm{iii}}$ & 0.93 & 2.59 & $3.289(4)$ & 132 \\
$\mathrm{C} 49 A-\mathrm{H} 49 A \cdots \mathrm{O} 12 A$ & 0.93 & 2.30 & $2.63(3)$ & 100 \\
$\mathrm{C} 2-\mathrm{H} 2 \cdots C g 2^{\mathrm{iv}}$ & 0.93 & 3.00 & $3.610(3)$ & 125 \\
$\mathrm{C} 15-\mathrm{H} 15 \cdots C g 8^{\mathrm{v}}$ & 0.93 & 2.99 & $3.665(4)$ & 131 \\
$\mathrm{C} 36-\mathrm{H} 36 \cdots C g 3$ & 0.93 & 2.81 & $3.487(3)$ & 130 \\
$\mathrm{C} 43 A-\mathrm{H} 43 A \cdots C g 4^{\mathrm{vi}}$ & 0.93 & 2.80 & $3.539(5)$ & 137 \\
$\mathrm{C} 43-\mathrm{H} 43 \cdots C g 4^{\mathrm{vi}}$ & 0.93 & 2.94 & $3.590(11)$ & 128
\end{tabular}

Symmetry codes: (i) $x, y-1, z$; (ii) $x, y-1, z+1$; (iii) $-x+1,-y+1,-z$; (iv) $-x+1,-y,-z$; (v) $-x+1,-y+1,-z+1$; (vi) $-x,-y+1,-z+1$. 